\title{
DNA methylome analysis of acute lymphoblastic leukemia cells reveals stochastic de novo DNA methylation in CpG islands
}

\begin{abstract}
Aim: To identify regions of aberrant DNA methylation in acute lymphoblastic leukemia (ALL) cells of different subtypes on a genome-wide scale. Materials \& methods: Wholegenome bisulfite sequencing (WGBS) was used to determine the DNA methylation levels in cells from four pediatric ALL patients of different subtypes. The findings were confirmed by 450k DNA methylation arrays in a large patient set. Results: Compared with mature B or T cells WGBS detected on average 82,000 differentially methylated regions per patient. Differentially methylated regions are enriched to $\mathrm{CpG}$ poor regions, active enhancers and transcriptional start sites. We also identified approximately $8000 \mathrm{CpG}$ islands with variable intermediate DNA methylation that seems to occur as a result of stochastic de novo methylation. Conclusion: WGBS provides an unbiased view and novel insights into the DNA methylome of ALL cells.
\end{abstract}

First draft submitted: 4 May 2016; Accepted for publication: 4 August 2016; Published online: 23 August 2016

Keywords: acute lymphoblastic leukemia $\bullet \mathrm{CpG}$ islands $\bullet$ DNA methylation $\bullet$ epigenome - methylome $\bullet$ whole-genome bisulfite sequencing

\section{Background}

DNA methylation at $\mathrm{CpG}$ dinucleotides is fundamentally important for cellular lineage commitment and memory [1]. Aberrant DNA methylation patterns are frequently observed in cancers, although the underlying mechanisms and the functional consequences of cancer related DNA methylation changes have remained elusive. The recent discovery of recurrent somatic mutations in key enzymes involved in the regulation of DNA methylation in hematologic malignancies suggests that disruption of the DNA methylation pattern can drive the transformation of normal cells to malignant states [2-4].

Acute lymphoblastic leukemia (ALL) comprises a genetically heterogeneous group of leukemias that originates from the transformation of common lymphoid progenitor cells into malignant cells, characterized by excessive proliferation of undifferentiated cells [5]. The precise molecular mechanisms that are involved in the transformation of lymphoid progenitor cells into ALL cells are currently unknown. Epigenetic changes may play an important role in the leukemic transformation in combination with somatically acquired mutations or other genetic aberrations. Using array-based DNA methylation analysis we and others have previously shown that DNA methylation patterns of ALL cells discriminate between cytogenetic subtypes of ALL and that the vast majority of the differential DNA methylation is enriched to CpG islands [6,7]. Furthermore, DNA methylation marks in ALL have also been associated with relapse [8], poor prognostics [9] and survival [10]. Historically, the focus of DNA methylation studies in cancer has been on CpG islands in gene promoter regions [11,12]. More recently, unbiased whole-genome bisulfite sequencing (WGBS) of normal tissues and cancer cells has highlighted the importance of distal regulatory enhancer elements
Per Wahlberg', Anders Lundmark', Jessica Nordlund', Stephan Busche2, Amanda Raine', Karolina Tandre ${ }^{3}$, Lars Rönnblom ${ }^{3}$, Daniel Sinnett ${ }^{4}$, Erik Forestier ${ }^{5}$ Tomi Pastinen², Gudmar Lönnerholm ${ }^{6} \&$ Ann-Christine Syvänen ${ }^{\star 1}$

${ }^{1}$ Department of Medical Sciences, Molecular Medicine \& Science for Life Laboratory, Uppsala University, Uppsala Sweden

${ }^{2}$ Department of Human Genetics, McGill University \& Genome Quebec Innovation Centre, Montreal, Quebec, Canada ${ }^{3}$ Department of Medical Sciences, Rheumatology, Uppsala University Uppsala, Sweden

${ }^{4}$ Research Center, Sainte-Justine University Health Center; Department of Pediatrics, University of Montreal, Montreal, Quebec, Canada ${ }^{5}$ Department of Medical Biosciences, University of Umeå, Umeå, Sweden ${ }^{6}$ Department of Women's \& Children's Health, Pediatric Oncology, Uppsala University, Uppsala, Sweden *Author for correspondence: ann-christine.syvanen@medsci.uu.se 
as regions of dynamic DNA methylation and functional relevance for cell specification and maturation [13-15]. Enhancer regions can be identified using patterns of DNA methylation as low levels of DNA methylation often coincide with transcription factor (TF) binding sites [16]. A WGBS study in pediatric medulloblastoma recently identified specific unmethylated genomic regions that distinguish between subtypes and are enriched for subtype-specific TF motifs [17].

In the current study we characterized the DNA methylomes of three pediatric B-cell precursor ALL (BCP-ALL) patients of different cytogenetic subtypes and one pediatric T-ALL patient using WGBS. In addition, we sequenced the transcriptomes of the ALL patients to assess the interplay between DNA methylation and gene expression. The results shed light on the DNA methylation patterns in ALL cells at diagnosis, and identify differential methylation in ALL cells compared with normal $\mathrm{B}$ and $\mathrm{T}$ cells.

\section{Material \& methods}

\section{Ethics statement}

The study was approved by the regional ethical review board in Uppsala, Sweden and the guardians of the pediatric ALL patients and the healthy subjects provided informed consent.

\section{Patients}

Swedish ALL patients (P1-P4) included in this study were diagnosed and treated according to the Nordic Society for Pediatric Hematology and Oncology (NOPHO) ALL2000 protocol [18]. The ALL patients (P1-P4) were carefully selected to be typical representatives of four major subgroups of childhood ALL based on unequivocal results from cytogenetic analyses. We reasoned that these four large cytogenetically defined subgroups of ALL should allow identification of DNA methylation patterns that are shared between ALL subtypes and patterns that are subgroup-specific. BCP-ALL with the $\mathrm{t}(12 ; 21)$ ETV6-RUNX1 gene fusion (P1) is the largest homogeneous cytogenetically defined subgroup of BCP-ALL representing 20-25\% of the patients with pediatric ALL. The patients with nonrecurrent (P3) or undetectable (P2) large-scale cytogenetic aberrations constitute two large subgroups of BCP-ALL. T-ALL (P4) represents $10 \%$ of the pediatric ALL cases. The patients P1-P4 have previously been whole-genome sequenced and are denoted: ALL-458 (P1), ALL-501 (P2), ALL-707 (P3) and ALL-559 (P4) in this study [19].

To compensate for the small size of our WGBS 'discovery cohort' with four ALL samples, we used data from a large cohort of approximately 700 ALL Nordic patients (and control cells) analyzed by $450 \mathrm{k}$
BeadArrays (Illumina) [6] to validate some of the observations made by WGBS.

For validation and control of differentially methylated regions (DMRs) discovered in the BCP-ALL patient (P1) with the $\mathrm{t}(12 ; 21)$ ETV6-RUNX1 subtype three samples (ALL_C1, ALL_C2 and ALL_C3) with the same ALL subtype from a Canadian cohort (Sainte-Justine Hospital, Montreal, Canada) were included in our study [20].

\section{Sample preparation \& DNA extraction}

Mononuclear cells from the ALL patients (P1-P4) were collected from bone marrow aspirates by FicollIsopaque (Pharmacia) density-gradient centrifugation at the time of diagnosis. The leukemic cell content of the samples exceeded $90 \%$ of the total cell content. DNA and total RNA were extracted from the ALL patient samples using reagents from the AllPrep DNA/RNA Mini Kit (QIAGEN). CD3 ${ }^{+} \mathrm{T}$ cells and $\mathrm{CD} 19^{+} \mathrm{B}$ cells were isolated from peripheral blood from healthy Swedish blood donors by positive selection using $\mathrm{CD}_{1}{ }^{+}$and $\mathrm{CD}^{+}$Microbeads and MACS cell separation reagents (Miltenyi). DNA and total RNA was extracted from the cells by the AllPrep DNA/RNA Mini Kit (QIAGEN). RNA concentrations were determined using Qubit (ThermoFisher) and the integrity of the RNA was verified by Agilent Bioanalyzer 2100 (Agilent technologies).

\section{Whole-genome bisulfite library preparation \& sequencing}

Whole-genome bisulfite sequencing libraries were prepared using the Illumina TruSeq DNA Library preparation kit. A total of $4 \mu \mathrm{g}$ of sample DNA together with $20 \mathrm{ng}$ of unmethylated lambda DNA (Promega) were fragmented by acoustic sonication (Covaris). Three parallel libraries were prepared from each sample starting with $1 \mu \mathrm{g}$ of DNA. End-repair, A-tailing and ligation of adaptors were performed according to the Illumina TruSeq protocol. Subsequent bisulfite conversion of the sequencing libraries was performed using reagents from the EpiTect Bisulfite kit (Qiagen). Bisulfite converted adaptor-ligated fragments were amplified by PCR in a reaction mixture containing $15-20 \mu \mathrm{l}$ of DNA template, $5 \mu \mathrm{l}$ Pfu Turbo Cx Reaction buffer (Stratagen), $5 \mu$ Illumina primer cocktail mix, $1.25 \mu \mathrm{l}$ dNTP mix $(10 \mathrm{mM}), 2.5$ units Pfu TurboCx Hot-start DNA polymerase (Stratagen) and $\mathrm{H}_{2} \mathrm{O}$ to a final volume of $50 \mu \mathrm{l}$. Cycling conditions during PCR were $5 \mathrm{~min}$ at $95^{\circ} \mathrm{C}$ and $30 \mathrm{~s}$ at $98^{\circ} \mathrm{C}$, followed by six cycles of 10 s at $98^{\circ} \mathrm{C}, 30$ s at $65^{\circ} \mathrm{C}, 30 s 72^{\circ} \mathrm{C}$, followed by $72^{\circ} \mathrm{C}$ for $5 \mathrm{~min}$. The bisulfite-converted sequencing libraries were subjected to paired-end $100 \mathrm{bp}$ sequencing on a HiSeq 2000 sequencing system (Illumina). 


\section{Alignment of bisulfite-converted sequence reads to reference genome}

Paired-end bisulfite converted reads from ALL patients and controls were mapped to the human reference genome (hg19) according to methods described by Johnson et al. [21]. Prior to aligning the sequence reads to the reference genome, adaptor sequences and bases with a low quality score $(<20)$ were removed using CutAdapt [22]. The reads were aligned to in silico bisulfite converted genomes using the Burrows-Wheeler aligner (BWA) [23]. Picard [24] was used to remove duplicated reads. In addition read pairs were removed if they did not map at the expected distance based on the average library insert size. Reads with a low mapping quality score $(<20)$ were also removed. Second, read pairs were removed if they mapped in the wrong orientation. Third, reads that mapped to both the forward and reverse converted reference strands were removed. Finally, reads were discarded if the mismatch rate to the converted reference exceeded $2 \%$ over an alignment length.

\section{DNA methylation calling}

The first and last three bases of each read-pair were removed to avoid biased methylation calls during the first and last three sequencing cycles due to introduction of unmethylated cytosines during end-repair. The Bis-SNP package (version 0.78) was used to determine the DNA methylation levels genome-wide [25]. To improve the precision of the methylation calls, the local realignment and base recalibration functions of Bis-SNP was applied. Raw calls were subsequently filtered using the Bis-SNP VCFpostprocess walker. Methylation values from the forward and reverse strands of individual $\mathrm{CpG}$ sites were combined into a single value and used in all downstream analysis. $\mathrm{CpG}$ sites that Bis-SNP called as heterozygous and CpG sites located on sex-chromosomes were removed from all downstream analysis. Low coverage bisulfite sequencing data from eight B-cell and 18 T-cell samples were combined into cell type-specific pools and methylation levels were calculated in the pools. The DNA methylome data (WGBS) from hematopoietic stem/progenitor cells $\left(\mathrm{CD} 34^{+} \mathrm{CD} 38^{\left.-\mathrm{Lin}^{-}\right)}\right.$were downloaded from GEO (accession no. GSM791828 [26]).

Average DNA methylation levels were calculated in 1-kb windows genome-wide for ALL P1-4, pooled $\mathrm{B}$ and $\mathrm{T}$ cells and of $\mathrm{B}$ and $\mathrm{T}$ cells from individuals with low coverage sequencing data that constitute the pooled B- and T-cell controls. To examine the effect of sequence read coverage on DNA methylation levels calculated in $1-\mathrm{kb}$ windows, the aligned reads from patient P4 was downsampled using Picards DownsampleSam to sequence coverages ranging from $3 \times$ to
$25 \times$, followed by recalling the DNA methylation levels using BisSNP.

\section{Statistical analysis of data \& visualization}

The statistical analyses and visualization of the data were performed using the $\mathrm{R}$ framework (version 3.2.2).

\section{Smoothing of DNA methylation data \& identification of differentially methylated regions}

The R-package bsseq [27] was used to smoothen methylation calls from WGBS data, segment data and call DMRs between sample pairs. To call a segment as a DMR a minimum of three consecutive CpG sites and an average difference $>0.3$ in the DNA methylation levels between an ALL sample and the control were required. BCP ALL samples (P1-P3) were compared with a pool of $\mathrm{B}$ cells (see above) and the T-ALL sample (P4) was compared with a pool of T cells.

To asses normal variation in $\mathrm{B}$ and $\mathrm{T}$ cells, eight individual B-cell samples and eight individual T-cell samples were compared with the remaining pooled B-cell and T-cell samples respectively, calling DMRs as described above. DMRs with an average mapability score (wgEncodeCrgMapabilityAlign100mer, download from UCSC table browser) of $<0.5$ across segments and DMRs where the average coverage in any of the compared pair of samples was less than three or larger than 50 (except in pooled samples), were removed to ensure that potential mapping errors did not inflate DMR detection.

Methylation levels of DMRs in ALL samples were considered to be shared with control cells if the average DMR methylation level of the other control cell was closer to the ALL sample than the control cell in the compared pair. When comparing methylation levels of DMRs between ALL cells, an average methylation difference of $<0.3$ for the DMRs was considered as similar. The degree of base overlap (defined by the Jaccard similarity index) between DMRs detected in ALL cells and control cells were computed using BEDTools [28].

\section{Principal component analysis}

Principal components of DNA methylation data from $\mathrm{CpG}$ sites in $\mathrm{CpG}$ island shores (defined as $2 \mathrm{~kb}$ on either side of a CpG island) were determined using the function prcomp in $\mathrm{R}$.

\section{Genomic annotation \& enrichment analysis of DMRs}

RefSeq and $\mathrm{CpG}$ island annotations were downloaded using the UCSC table browser (GRCh37/hg19, track RefSeq Genes, table refGene and RefSeq CpG Islands, table cpgIslandExt) to define exons, introns, UTR5, 
TSS1500 and intergenic regions. CpG island shores were defined as $2 \mathrm{~kb}$ of sequence flanking a $\mathrm{CpG}$ island. Each base in the DMRs was assigned to a genomic feature, and the number of bases in the individual features was divided with the total number of bases defined as a DMR. Sample specific genomic backgrounds were derived by intervals of $\mathrm{CpG}$ sites with sufficient read coverage no more than $2 \mathrm{~kb}$ apart. The genomic background provides a set of intervals where DMRs can theoretically be called and the genomic background was used to compute an enrichment score for each genomic feature.

\section{Evolutionary conservation in DMRs}

PhyloP base-wise score derived from alignment of 46 vertebrate species were downloaded (UCSC table browser) and used to infer evolutionary conservation in DMR sequences. The PhyloP score profiles along DMRs were calculated by binning the genomic range of each DMR with flanks of the same length as the DMR into 50 equal sized genomic ranges. The average PhyloP score of each bin was calculated and then averaged per bin across all DMRs in each sample. As a general control of conservation we used a size matched set of fragments randomly chosen from regions in the genome where the $\mathrm{CpG}$ sites are positioned sufficiently close to each other to be called as a DMR using the DMR detection settings described above.

\section{Functional annotations \& enrichment of DMR to chromatin states}

Chromatin state maps generated by the Roadmap Epigenomics project [29] were used to ascertain genomic positions and infer functions of DMRs. An 18-state chromatin map constructed from the six histone marks (H3K4me3, H3K4me1, H3K36me3, H3K27me3, $\mathrm{H} 3 \mathrm{~K} 9 \mathrm{me} 3$ and H3K27ac) was downloaded [30] for B cells (Epigenome ID: E032), T cells (Epigenome ID: E034) and CD34+ cells (Epigenome ID: E050). Foldenrichment of DMRs to each state was calculated by computing the proportion of bases overlapping with each chromatin state, followed by division by the genome-wide proportions of bases in different chromatin states. Significance thresholds for enrichment were set by empirical $\mathrm{p}$-values determined by permutation testing. DMRs were randomly shuffled 1000-times within the theoretical genomic intervals described earlier using BEDTools [28].

\section{TF binding \& motif enrichment analysis}

Chromatin immunoprecipitation sequencing data from the ENCODE project [31] was used to obtain information about TF binding sites for 161 TFs. Enrichment of TF binding motifs to DMRs was calculated as described by Kheradpour and Kellis [32] and implemented in the program ENCODE-motifs [33]. Hierarchical clustering of a motif similarity matrix (downloaded from [34]) was performed in order to describe the relationship between TF binding sites motifs and used to order TFs into groups.

\section{Gene-expression analysis}

Ribosomal RNA was depleted from $1 \mu \mathrm{g}$ total RNA using Ribo-Zero (Illumina) and strand-specific RNAsequencing libraries were constructed with the ScriptSeq V2 Kit (Epicentre). Libraries were sequenced with either 100 or 50 bp sequencing chemistry (paired-end) on a HiSeq2000/2500 sequencing instrument (Illumina). Reads were mapped to the reference genome (human_g1k_v37) using Tophat2 (version 2.0.4) with default settings [35]. The number of fragments per transcript was calculated from Tophat bam files using the function featureCounts in Rsubread R-package [36] and fragments per transcript per kilobase per million was computed by the function rpkm in the package edgeR [37]. When used in differential expression analysis between ALL cells and B and T cells, transcript fragment counts were normalized to $\log _{2}$ counts using the voom function in the limma R-package [38]. A linear model was fitted to the data to calculate differences in expression level and p-values corrected for multiple testing were determined using the eBayes function in limma. We considered a transcript as differentially expressed between samples if the $\log _{2}$ fold-change was $>1$ and p-value $<0.01$. DMRs were assigned to a protein-coding transcript (RefSeq) if a DMR was located within $10 \mathrm{~kb}$ upstream of a transcription start site to $5 \mathrm{~kb}$ downstream of a protein-coding transcript.

\section{Gene ontology term analysis of CpG islands}

We used Genomic Regions Enrichment of Annotations Tool (GREAT) [39] to investigate enrichment of biological processes and phenotypic associations to CpG islands.

\section{Analysis of heterogeneity of $\mathrm{CpG}$ island methylation in ALL cells}

DNA methylation patterns within individual sequence reads were used to examine $\mathrm{CpG}$ islands with intermediate DNA methylation levels. Read-pairs with less than five CpG sites and lower than an average $5 \times$ coverage across a $\mathrm{CpG}$ island were excluded from the analysis. The read-pairs were categorized into concordantly methylated (either unmethylated or methylated) if $90 \%$ of the CpG sites along a read-pair showed the same methylation state or as discordantly methylated if a read-pair contained at least $10 \%$ of either methylated or unmethylated CpG sites. 
The variance between sequence reads was calculated as follows: $\mathrm{CpG}$ sites in a $\mathrm{CpG}$ island were recoded with 0 representing unmethylated and 1 representing methylated states. For each $\mathrm{CpG}$ site in a $\mathrm{CpG}$ island the sum of the squared differences between cytosine methylation levels and the CpG site average methylation level $S S_{s}$ were calculated according to the following formula:

$$
\mathrm{SS}_{\mathrm{s}}=\sum_{\mathrm{c}=1}^{\mathrm{n}}\left(\mathrm{x}_{\mathrm{c}}-\overline{\mathrm{x}_{\mathrm{s}}}\right)^{2}
$$

Where $n$ is the number of reads covering the CpG site $s$.

$\overline{\mathrm{X}_{s}}$ is average methylation level of the CpG site $s$.

$X_{c}$ is the methylation status ( 0 or 1 ) of a CpG site $s$.

The sum of the squared differences for each $\mathrm{CpG}$ site is then summed across each $\mathrm{CpG}$ island and divided by the total number of $\mathrm{CpG}$ sites in the $\mathrm{CpG}$ island minus one.

$$
\frac{\sum_{s=1}^{\mathrm{k}} \mathrm{SS}_{\mathrm{s}}}{\left(\sum_{\mathrm{s}=1}^{\mathrm{k}} \mathrm{n}_{\mathrm{s}}\right)-1}
$$

Where $k$ is the number of $\mathrm{CpG}$ sites in the CpG island. $S S_{s}$ is the sum of squared differences in $\mathrm{CpG}$ site $s$. $n$ is the number of reads covering CpG site $s$.

\section{Results}

\section{Data \& global patterns of DNA methylation} in ALL

We bisulfite sequenced the ALL genomes of four patients (patients P1-P4) in bone marrow samples collected at diagnosis, to an average 20-30-fold sequence depth per base (Table 1 \& Supplementary Table 1). At this sequence depth between 90 and $93 \%$ of the CpG sites in the ALL genomes are covered by at least five sequencing reads. Patient 1 has the recurrent BCPALL translocation $\mathrm{t}(12 ; 21)$ ETV6-RUNX1, Patient 2 has BCP-ALL with two nonrecurrent translocations, Patient 3 has BCP-ALL with a normal karyotype and Patient 4 has T-cell immunophenotype ALL (T-ALL). The concordance between the DNA methylation levels determined by WBGS and by Infinium HumanMethylation 450 arrays (450k BeadArray) from the same patient samples [6] ranged between 0.97 and $0.98(\mathrm{r})$ per patient (Supplementary Figure 1A \& B). In the WGBS data from the four ALL samples we did not find evidence of DNA methylation at other sites than CpG dinucleotides. The genome-wide DNA methylation levels for individual CpG sites are largely bimodally distributed, with the majority of $\mathrm{CpG}$ sites either unmethylated or completely methylated in the ALL samples (Supplementary Figure 2). Using a method described by Burger et al. [16] to identify segments of continuous hypomethylation, we did not find evidence of large megabase-scale partially methylated domains in the ALL patients like those observed in colon cancer [40] and medulloblastoma cells [17].

We first explored the global DNA methylation patterns by determination of the median DNA methylation levels in $1 \mathrm{~kb}$ windows across the four ALL genomes and genomes of pooled B cells $(n=8)$ and $T$ cells $(n=17)$ from healthy blood donors. We observed an average approximately 5\% increase in global DNA methylation levels in ALL cells compared with $B$ and T cells $\left(\mathrm{p}<2.2 \times 10^{-16}\right)$ (Figure $\left.1 \mathrm{~A}\right)$. We also observed individual variation in genome-wide median CpG methylation levels between the ALL patients and between the low-coverage $\mathrm{B}$ and $\mathrm{T}$ cells from the individual donors. To verify that the results from lowcoverage $\mathrm{B}$ and $\mathrm{T}$ cells are comparable with the data from ALL P1-4 we downsampled data from the T-ALL (P4) patient to $3 \times$ sequence coverage. We observed marginal differences in median DNA methylation levels in $1 \mathrm{~kb}$ windows across the genome in the range from $25 \times$ to $3 \times$ coverage (Supplementary Figure 3 ). The individual variation in genome-wide $\mathrm{CpG}$ methylation levels is supported by $450 \mathrm{k}$ BeadArray data from a large number of ALL patients $(\mathrm{n}=550)$ [6] with different

Table 1. Clinical information and sequencing statistics for acute lymphoblastic leukemia samples included in this

\begin{tabular}{|c|c|c|c|c|c|}
\hline Patient ID ${ }^{\dagger}$ & Genetic subtype & $\begin{array}{l}\text { Immune } \\
\text { phenotype }\end{array}$ & Sex:age (years) & $\begin{array}{l}\text { Average } \\
\text { coverage }^{\pi}\end{array}$ & $\begin{array}{l}\text { Bisulfite conversion } \\
\text { rate }(\%)^{\#}\end{array}$ \\
\hline P1 (ALL_458) & $\mathrm{t}(12 ; 21)$ ETV6-RUNX1 & BCP-ALL & Male:3.6 & $20 x$ & 99.97 \\
\hline P3 (ALL_707) & Other & BCP-ALL & Male:1.6 & $29.5 x$ & 99.97 \\
\hline P4 (ALL_559) & T-ALL & T-ALL & Male:5.9 & $28 x$ & 99.73 \\
\hline
\end{tabular}
study. 


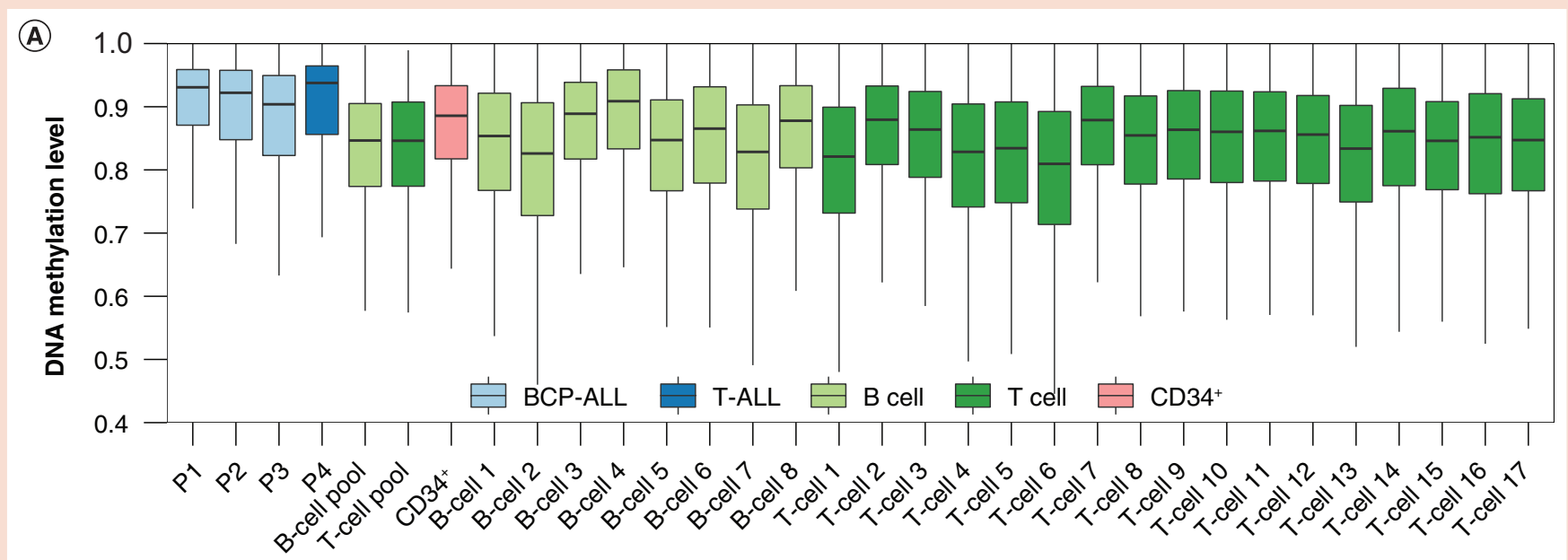

(B)
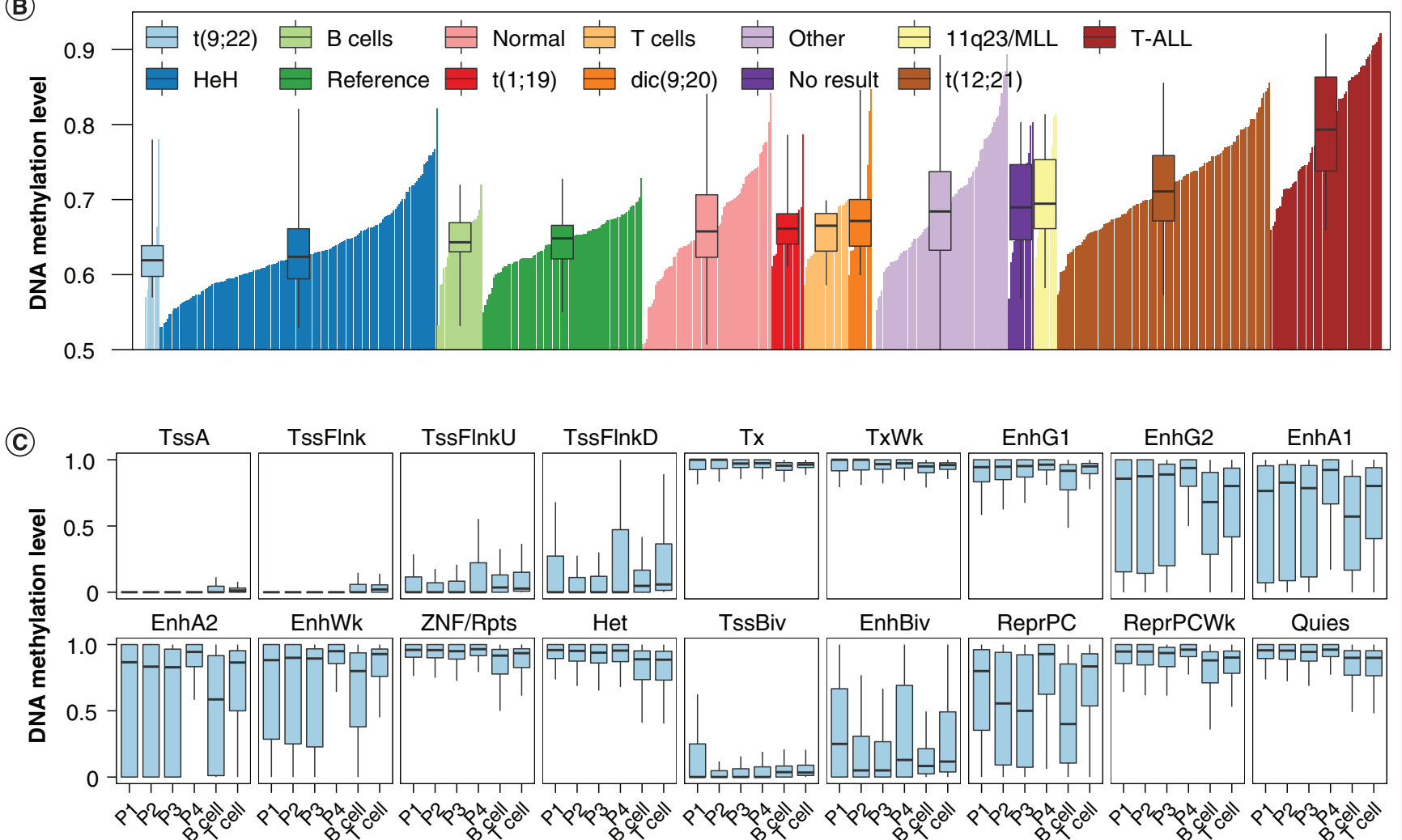

Figure 1. Global DNA methylation levels in acute lymphoblastic leukemia cells and in normal B and T cells (see facing page).

(A) Box-plots showing DNA methylation levels of cytosines in CpG sites averaged within 1-kb windows across the genome. Average DNA methylation levels were calculated in $1 \mathrm{~kb}$ windows from individual ALL patient samples (P1-P4), pooled B and T cells, individual B-cell and T-cell control samples and hematopoietic stem cells (CD34+). The horizontal line in box-plots shows median values. ALL: Acute lymphoblastic leukemia; BCP-ALL: B-cell precursor ALL; EnhG1: Genic enhancer; EnhG2: Genic enhancer; EnhA1: Enhancer; EnhA2: Enhancer; EnhBiv: Bivalent enhancer; EnhWk: Weak enhancer; Het: Heterochromatin; Quies: Quiescent or low expression; ReprPC: Repressed polycomb; ReprPCWk: Weakly repressed polycomb; TssA: Active transcription start site; TssBiv: Bivalent/ poised transcription start site; TssFlnk: Flanking active transcription start site; TssFlnkD: Flanking downstream active transcription; TssFlnkU: Flanking upstream active transcription; Tx: Strong transcription; TxWk: Weak transcription; ZNF/Rpt: ZNF gene and repeat.

cytogenetic subtypes and control blood cells $(n=139)$, where the median DNA methylation levels across the sites on the array show extensive heterogeneity ranging from 0.51 to 0.92 (Figure 1B). The high variability in DNA methylation appears to be independent of ALL subtype, although the T-ALL samples consistently dis- 
Figure 1. Global DNA methylation levels in acute lymphoblastic leukemia cells and in normal B and T cells (cont.). (B) Bar-plot of genome-wide median DNA methylation levels from 450k Bead Arrays. The inserted box-plots show median DNA methylation levels and variation in methylation levels for each ALL subtype. Subtypes and control cells are sorted in ascending order according to median DNA methylation levels within each subtype group and are color coded. The subtype annotated as 'reference' refers to ALL patients that are in remission, 'normal' corresponds to an ALL subtype with a normal karyotype, 'no results' to ALL patients that failed karyotyping and 'other' corresponds to ALL subtype that have a none-reoccurring karyotype. (C) DNA methylation levels in ALL cells and control B and T cells at genomic positions defined by 18-state chromatin maps retrieved the Roadmap Epigenomics consortium. Data in boxes correspond to DNA methylation levels from individual CpG sites in ALL cells and pooled control B and T cells that overlap with the individual chromatin states. Horizontal bars correspond to median values. The analyzed samples are indicated on the X-axis in the lower panel and the designation of chromatin states are shown above the individual box-plots. Data from BCP-ALL patients P1-P3 and pooled control B cells were compared with B-cell chromatin state maps. Data from the T-ALL patient P4 and pooled control T cells were compared with T-cell chromatin states maps.

ALL: Acute lymphoblastic leukemia; BCP-ALL: B-cell precursor ALL; EnhG1: Genic enhancer; EnhG2: Genic enhancer; EnhA1: Enhancer; EnhA2: Enhancer; EnhBiv: Bivalent enhancer; EnhWk: Weak enhancer; Het: Heterochromatin; Quies: Quiescent or low expression; ReprPC: Repressed polycomb; ReprPCWk: Weakly repressed polycomb; TssA: Active transcription start site; TssBiv: Bivalent/ poised transcription start site; TssFlnk: Flanking active transcription start site; TssFInkD: Flanking downstream active transcription; TssFInkU: Flanking upstream active transcription; Tx: Strong transcription; TxWk: Weak transcription; ZNF/Rpt: ZNF gene and repeat.

played the highest DNA methylation levels in the 450k BeadArray data.

We took advantage of chromatin state maps for $\mathrm{B}$ and $\mathrm{T}$ cells generated by the Epigenomics Road Map project [41] to examine the relationship between 18 reoccurring combinations (states) [42] of histone modifications and levels of DNA methylation in the ALL cells (Figure 1C). Expectedly, CpG sites overlapping with states annotated as active transcription start sites (TSS) show the lowest levels of DNA methylation, whereas CpG sites that map to 'gene bodies' in actively transcribed genes have the highest DNA methylation levels. This analysis also shows that the $\mathrm{CpG}$ sites located to active enhancer and Polycomb repressed chromatin states show large variability in DNA methylation levels in the ALL cells.

\section{Differential DNA methylation in low-density CpG regions in ALL cells}

To comprehensively define DMRs between ALL cells and nonleukemic cells, we compared the individual BCP-ALL patients (P1-P3) to pooled B cells and the T-ALL patient (P4) to pooled T cells. For calling a genomic region as a DMR, we required a minimum of three $\mathrm{CpG}$ sites per DMR, more than $3 \times$ sequencing coverage for each $\mathrm{CpG}$ site, and an average absolute difference $>0.3$ in the DNA methylation levels between an ALL sample and the control pool. Using these stringent criteria for calling DMRs, we identified between 74,267 and 103,275 DMRs in the three BCP-ALL patients (P1-P3) and 69,849 DMRs in the T-ALL patient (P4) (Table 2 \& Supplementary Table 2 [GSE76270]). The contribution by normal variation between the B-and T-cell samples in the pooled B and $T$ controls to the number of DMRs detected in the ALL samples was estimated to be between 4529 and 27,603 DMRs for B cells and between 2184 and 7947 DMRS for the T cells. Thus the contribution by normal variation to the number of DMRs detected in the ALL cells appears to be minor. Due to the fact that the data from the $B$ and $T$ cells have low sequence coverage, the accuracy of the analysis is lower than for the ALL samples with high coverage. The BCPALL patients P2 and P3 have an excess of hypomethylated DMRs, with a ratio of hyper- to hypo-methylated DMRs of 0.78 and 0.64 , respectively, whereas the BCP-ALL patient P1 shows a balanced number of hyper- to hypomethylated DMRs. On the contrary, the T-ALL sample P4 shows a large excess of hyper-methylated DMRs (ratio 2.7) (Table 2).

We validated the DNA methylation levels in DMRs by comparing the methylation levels determined by WGBS within each DMR against $\beta$-values obtained from 450k BeadArrays. Only approximately 20\% of the DMRs identified by WGBS are supported by at least one probe on the $450 \mathrm{k}$ BeadArray. For these DMRs we observe correlation values (r) between 0.87 and 0.93 (Supplementary Figure 4A). The remaining approximately $80 \%$ of the DMRs are not covered by any probe on the $450 \mathrm{k}$ BeadArray and only $0.2-1.6 \%$ of DMRs are covered by more than five probes (Supplementary Figure 4B). The limited overlap between DMRs identified by WGBS and 450k BeadArrays highlights the advantage of WGBS to discover DMRs across the genome.

The DMRs cover on average $31 \mathrm{Mb}$ per ALL genome (range: 24-42 Mb), which corresponds to approximately $1 \%$ of the genome. The number of CpG sites located within the DMRs in each patient varies between 536,237 and 1,011,619 (Table 2). The median number of $\mathrm{CpG}$ sites per DMR across all comparisons ranges between 5 and 6 , which implies that the DMRs are predominantly located in regions with a low-density of CpG sites. The majority of the DMRs in the ALL samples (74-81\%) are less than $500 \mathrm{bp}$ in size, and only a small minority of them $(0.4-1.4 \%)$ are larger than $2 \mathrm{~kb}$ (Figure $2 \mathrm{~A}$ ). The CpG sites located centrally within a DMR display 


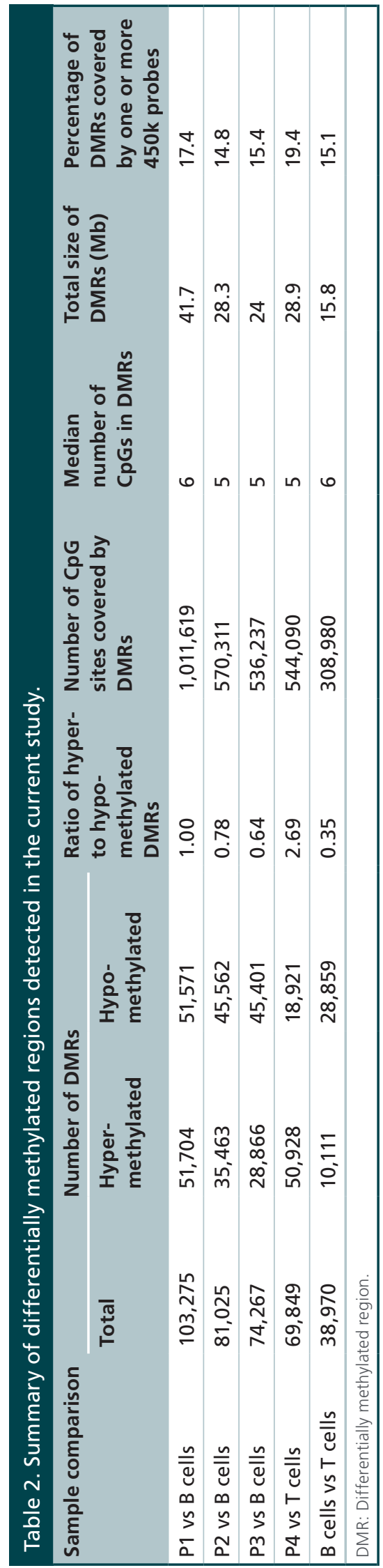

a larger degree of differential methylation than distally located $\mathrm{CpG}$ sites (Figure 2B), implying that the central part of a DMR plays a particularly important functional role.

Approximately $70 \%$ of the DMRs are unique for the ALL samples, while the remaining approximately $30 \%$ are shared with $\mathrm{CD} 34^{+}$cells, B cells and/or $\mathrm{T}$ cells (Figure 3A). Of the ALL-specific DMRs that are not present in control cells, between 63 and $83 \%$ are shared between the ALL cells (Figure 3B).

In low-coverage WGBS data from three additional patients with the $\mathrm{t}(12 ; 21)$ ETV6-RUNXI BCP-ALL subtype represented by patient P1 in our study we observe a similar number of DMRs (range: $62,210-56,327$ ) that overlap (Jaccard similarity index 0.23-0.34) with the DMRs in BCP-ALL patient P1 (Supplementary Figure 5A). Not surprisingly the correlation in genome-wide DNA methylation levels between the four t(12;21) ETV6-RUNX1 samples subjected to WGBS is lower than that between $163 \mathrm{t}(12 ; 21)$ ETV6-RUNX1 samples analyzed by the 450k BeadArrays [6] that targets a 60-fold lower number of $\mathrm{CpG}$ sites in selected $\mathrm{CpG}$-rich genomic regions. Principal component analysis of DNA methylation levels at CpG sites located in CpG island regions shows a strong subtype specific clustering of the ALL samples P1 and C1-3 (Supplementary Figure 5B). Figure 4A shows an example of a large DMR region that harbors two adjacent hypomethylated DMRs that were identified in each of the four BCP $t(12 ; 21)$ patients. The DMR region spans $19 \mathrm{~kb}$ and maps to the TERF2 (also known as TRF2). TERF2 is involved in the regulation of telomere length, and over-expression of TERF2 in cancer cells prevents the elimination of the cancer cells by natural killer cells [43]. Notably, the large DMR that spans the TERF2 gene contains only one probe on the 450k BeadArray, which explains why this large DMR has not previously been observed. According to RNA-sequencing data from ALL patients $(\mathrm{n}=125)$ of different ALL subtypes, the TERF2 locus shows elevated expression in a large proportion of BCP-ALL $\mathrm{t}(12 ; 21)$ patients compared with ALL patients with other subtypes and control $\mathrm{B}$ and $\mathrm{T}$ cells (Wilcoxon; $\mathrm{p}=4.79 \times 10^{-7}$; Figure $\left.4 \mathrm{~B}\right)$. The subtype-specific functional role of the TERF2 locus in $\mathrm{t}(12 ; 21)$ BCP-ALL warrants further studies of telomere function in this subtype.

\section{Enrichment of hematological TF binding sites to DMRs in ALL cells}

We annotated the DMRs to gene and CpG island regions and tested for an enrichment of DMRs to these genomic regions (Figure 5A). The DMRs are underrepresented in intergenic regions and enriched to gene 
(A)

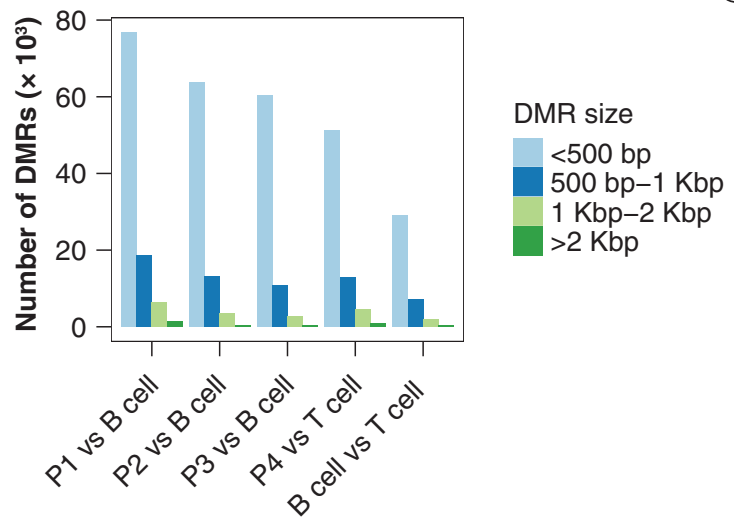

(B)

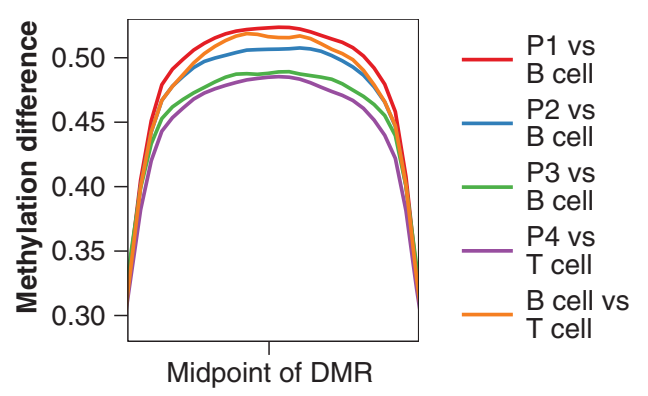

Figure 2. Size distribution and DNA methylation levels across differentially methylated regions. (A) Size distribution of DMRs. DMRs in B-cell precursor ALL patients (P1, P2 and P3) were called by comparison with B cells, and DMRs in T-ALL were called by comparison with T cells, as indicated on the X-axis. The DMRs are categorized into four color-coded groups according to their size as shown to the right of panel A. The height of the bars shown on the $\mathrm{Y}$-axis corresponds to the number of DMRs in each group. (B) Differences in average absolute methylation levels across DMRs. The width of the DMRs from individual comparisons was scaled by dividing them into 50 fractions and for each fraction an average methylation difference was calculated. The key to the right of panel B shows the five pair-wise cell comparisons to call the DMRs that are included in the plot.

DMR: Differentially methylated region.

regions. DMRs in CpG island shores show an equal distribution of hyper- and hypomethylated DMRs. Hyper-methylated DMRs are approximately three- to fourfold over-represented in CpG islands and 'shores' in the ALL samples, with the exception of patient P1 with $\mathrm{t}(12 ; 21)$ ETV6-RUNX1 BCP-ALL where hypermethylated DMRs are as much as eightfold enriched to CpG islands. The nucleotide sequences of the DMRs show stronger evolutionary conservation (PhyloP) than the regions immediately flanking the DMRs, suggesting that functional DNA sequences in the ALL cells are enriched in DMRs (Supplementary Figure 6). To increase the resolution of the annotation of DMRs, we used chromatin state maps from normal B-, T cells and $\mathrm{CD} 34^{+}$cells (Figure 5B) [41]. The DMRs in ALL cells are enriched to regions predicted to be enhancer elements or TSS, whereas DMRs are underrepresented in regions marked as actively transcribed, containing repeats, silenced and as heterochromatin. As has been observed previously in ALL [6], hyper-methylated DMRs in ALL samples are over-represented in bivalently marked TSS and enhancer states and to regions repressed by polycomb proteins (ReprPC). Chromatin immunoprecipitation sequencing (ChIP-Seq) data for 161 TFs from multiple cell types (ENCODE project [44]), show that a third (32-35\%) of the DMRs in the ALL cells overlap with at least one annotated TF binding site. To identify TFs of potential importance for ALL we scanned the DMRs against a database of approximately 2000 known and predicted
TF binding motifs [32]. As expected, we observe an over-representation of blood cell-specific TF binding motifs in the DMRs in the ALL cells (Figure 5C). The enrichment of TF binding motifs to hypo- and hypermethylated DMRs displays distinct patterns in ALL cells. Hypomethylated DMRs in BCP-ALL are, for example, enriched for PAX5 and ERG motifs, while hyper-methylated DMRs in all of the samples show an over-representation of NF- $\mathrm{kB}$ (NF- $\mathrm{kB}$-light chain enhancer of activated B cells) binding motifs. PAX5 plays an important role in B-cell differentiation [45], and is somatically mutated in approximately $30 \%$ of pediatric BCP-ALL cases [46], while NF- $\mathrm{KB}$ plays a central role in B-cell activation [47]. Thus DMRs could serve as footprints to detect previously unknown TFs and pathways that are important for ALL development and progression.

\section{Features of DMRs associated with differentially expressed genes}

Next we interrogated potential effects of DMRs on gene expression using RNA-sequencing data from three of the ALL patients (P1, P2, and P4) and B and $\mathrm{T}$ cells, for comparison (ALL patient $\mathrm{P} 3$ is not included here due to lack of RNA-sequencing data). Approximately $50 \%$ of the annotated protein coding genes are expressed (fragments per transcript per kilobase per million [FPKM] >1) in each ALL sample, and of them 880-1447 genes are differentially expressed between ALL cells and control B or T cells (defined as $\mathrm{p}<0.01$, 
(A)

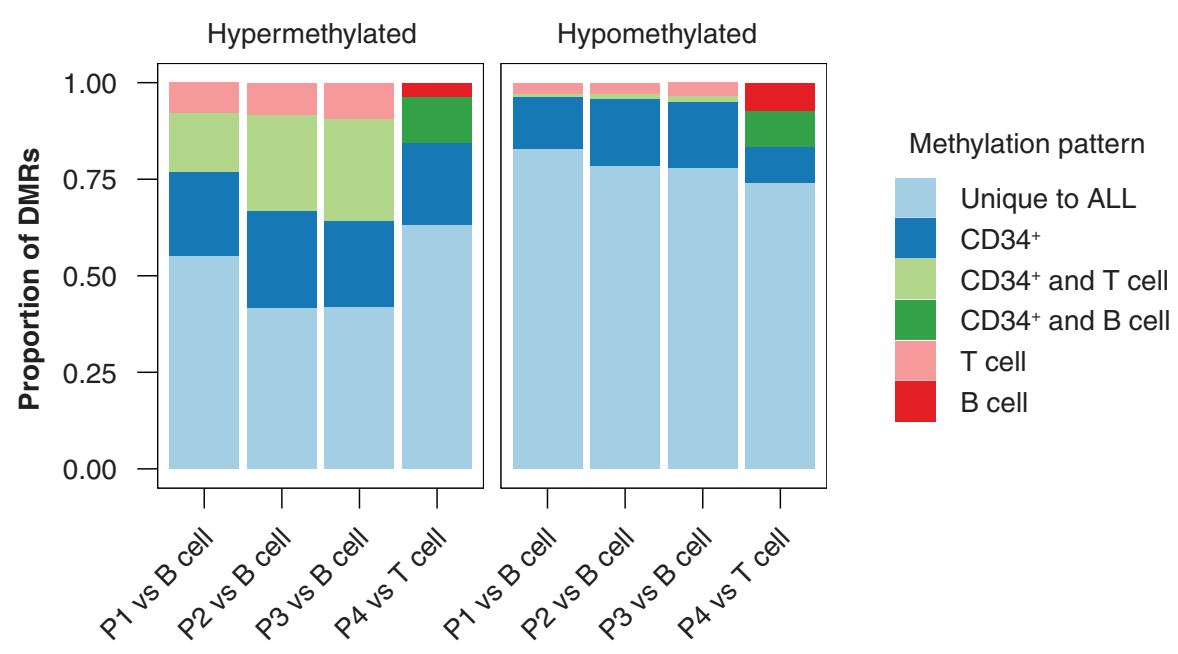

(B)

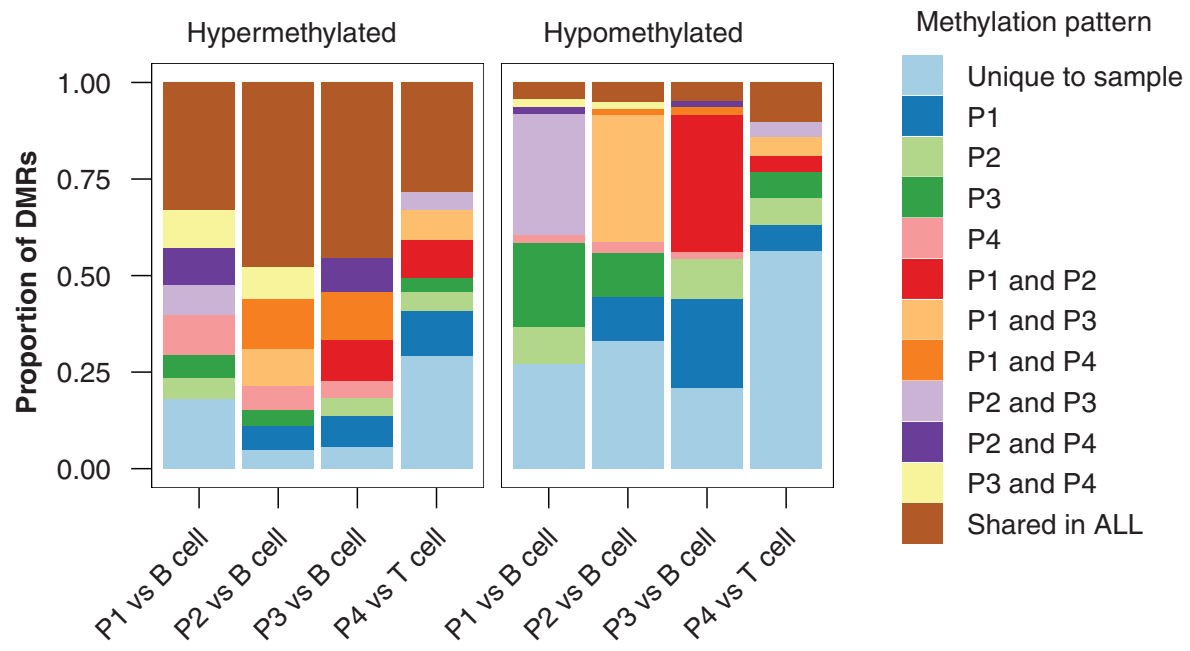

Figure 3. Proportions of differentially methylated regions shared between acute lymphoblastic leukemia cells and $\mathrm{B}, \mathrm{T}$ and $\mathrm{CD} 34^{+}$cells. Proportions on the Y-axes show the number of DMRs that are shared among ALL samples and control cells, calculated separately for hyper- and hypomethylated DMRs. Each column represents DMRs detected by comparing ALL cells from BCP-ALL patients P1-P3 to B cells and T-ALL patient P4 to T cells. The key on the right of the figures shows which cell type(s) share DMRs with ALL cells from ALL patients P1-P4. Hypermethylated DMRs have increased methylation in an ALL sample compared with the control cells, hypomethylated DMRs have decreased methylation in an ALL sample compared with control cells. (A) Proportions of DMRs that are unique to ALL cells or shared with one or more of the control cells. (B) Proportions of DMRs that are shared between one or more of the ALL samples, but that do not share DMRs with any control cells. ALL: Acute lymphoblastic leukemia; DMR: Differentially methylated region.

fold-change $>2$; Supplementary Table 3). Of the differentially expressed genes, 108 genes are shared between all three ALL samples, while a total of 1770 genes are differentially expressed in one of the ALL samples. One or more of the DMRs are co-localized to 9146-4409 protein coding genes and we observe an enrichment of DMRs to differentially expressed genes, compared with DMRs that are not located proximal to a differentially expressed gene (Fisher's Exact test; $\left.\mathrm{OR}=2-2.9 ; \mathrm{p}=1.25 \times 10^{-15}-2.2 \times 10^{-16}\right)$. Consistently,
DMRs associated with differentially expressed genes in ALL cells are on average larger (median: $310 \mathrm{bp}$ vs 231 bp; $\mathrm{p}<2.2 \times 10^{-16}$ ), the difference in absolute DNA methylation levels at the DMRs is higher (median: 0.390 vs $0.379 ; \mathrm{p}=1.52 \times 10^{-19}$ ) and a larger relative number of DMRs are associated with individual differentially expressed genes (median: 0.053 vs 0.041 ; $\left.\mathrm{p}=9.1 \times 10^{-81}\right)$, than DMRs associated with genes that do not display differential expression between ALL cells and normal blood cells (Figure 6A-D). 
DMRs that are located in the vicinity of differentially expressed genes are over-represented 2.4-7.7-fold to active TSS states, compared with DMRs annotated to genes that do not display differential expression (Supplementary Figure 7).

\section{Heterogeneity of DNA methylation at CpG islands in ALL cells}

As we observed an enrichment of DMRs to CpG islands in the ALL cells (Figure 5A), we investigated the DNA methylation patterns at the CpG islands in greater detail. Pair-wise comparison of the DNA methylation levels in the $\mathrm{CpG}$ islands in ALL cells with normal B and T cells identified approximately 4000 differentially hyper-methylated CpG islands in ALL P1 $\mathrm{t}(12 ; 21)$, with an absolute mean difference larger than 0.2 , while in the three other ALL patients (P2, P3 and P4), $2000 \mathrm{CpG}$ islands are differentially hyper-methylated (Figure 7). The CpG islands partitioned into three groups based on their mean methylation levels. Consistently unmethylated or lowly methylated $\mathrm{CpG}$ islands (methylation levels between 0 and $15 \%$; mean $=1.8$; $\mathrm{SD}=2.5)$ constitute approximately $45 \%$ of the anno- tated $\mathrm{CpG}$ islands, $\mathrm{CpG}$ islands with high methylation levels (between 85 and 100\%; mean $=95.0 ; \mathrm{SD}=2.7$ ) constitute $20 \%$ of all CpG islands, while CpG islands with intermediate and highly variable methylation levels (mean $=37.5 ; \mathrm{SD}=31.3$ ), constitute $35 \%$ of all CpG islands (Figure 8A \& Supplementary Table 4). Interestingly $\mathrm{B}$ and $\mathrm{T}$ cells also show increased methylation at variable intermediately methylated $\mathrm{CpG}$ islands, although at significantly lower levels than the ALL cells (Supplementary Figure 8). DNA methylation data from 450k BeadArrays from a larger number of ALL samples $(n=550)$ confirm this pattern of highly variable and increased DNA methylation levels at $\mathrm{CpG}$ sites within CpG islands in ALL samples (Figure 8B).

According to gene ontology term analysis, intermediately methylated $\mathrm{CpG}$ islands are enriched to genes that are expressed in early development and to homeobox genes (Supplementary Table 5). As many as $45 \%$ of the $\mathrm{CpG}$ islands with intermediate methylation levels are co-localized with chromatin regions marked with bivalent histone marks and repressive polycomb proteins in B cells, contrary to unmethylated $\mathrm{CpG}$ islands, where only $13 \%$ overlap with

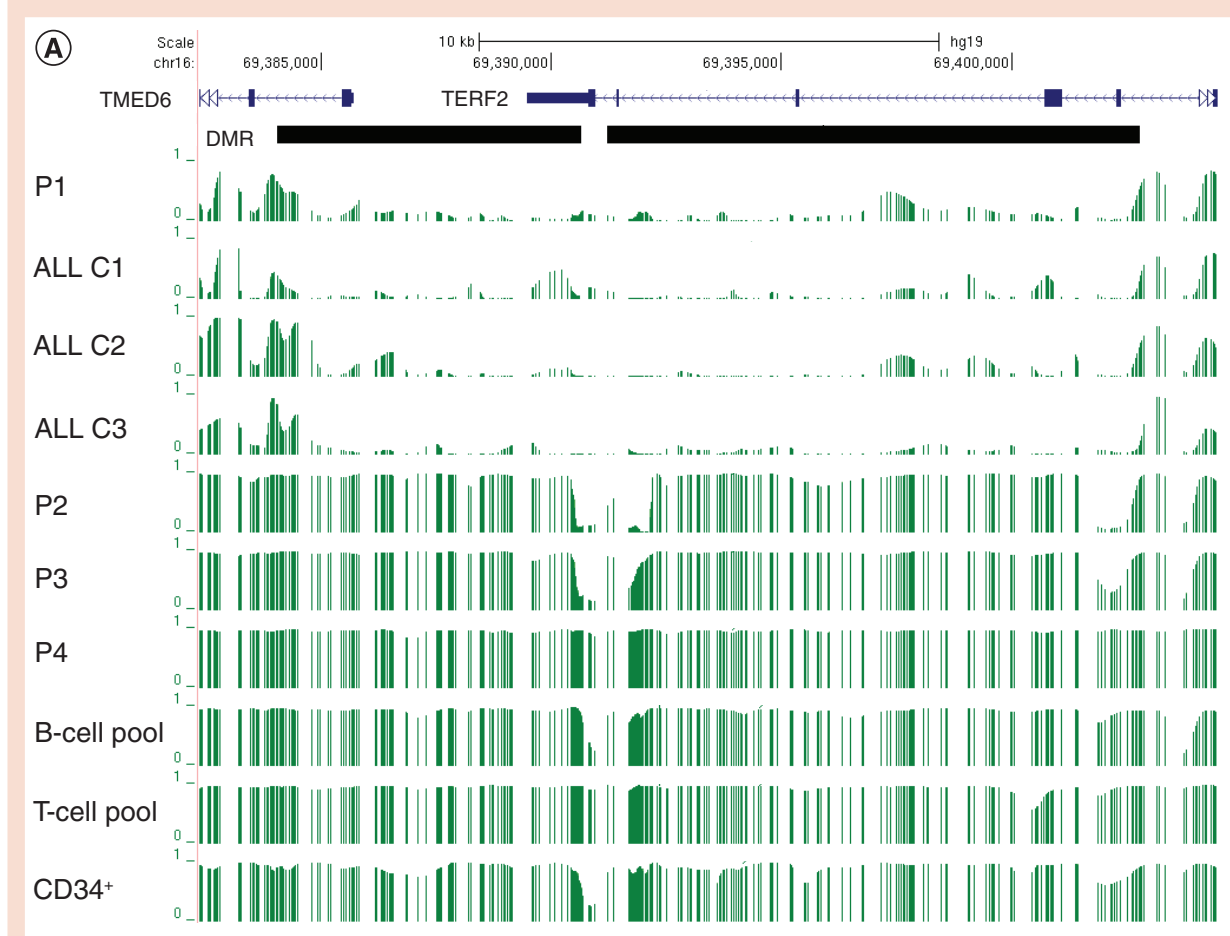

Figure 4. A $19 \mathrm{~Kb}$ differentially methylated region spanning the TERF2 gene discovered in the BCP-ALL t(12;21) ETV6-RUNX1 subtype. (A) Smoothed DNA methylation levels across two adjacent DMRs that cover a large part of the TERF2 locus. DNA methylation levels ranging from 0 to 1 as indicated on the vertical axes are shown for BCP-ALL t(12;21) ETV6-RUNX1 samples (P1, ALL-C1, ALL-C2, ALL-C3), BCP-ALL samples (P2, P3), the T-ALL sample (P4) and control B, T and CD34+ cells. (B) Expression levels (fragments per transcript per kilobase per million values) for the TERF2 gene in ALL samples of different subtypes and control cells. p-value (Wilcoxon rank sum test) for difference between expression level of the BCP-ALL $t(12 ; 21)$ subtype against the ALL samples $(n=105)$ of all other subtypes with the ALL subtypes indicated on the horizontal axis. The numbers below the box-plots indicate the number of samples in each subtype. ALL: Acute lymphoblastic leukemia; BCP-ALL: B-cell precursor ALL; DMR: Differentially methylated region. 


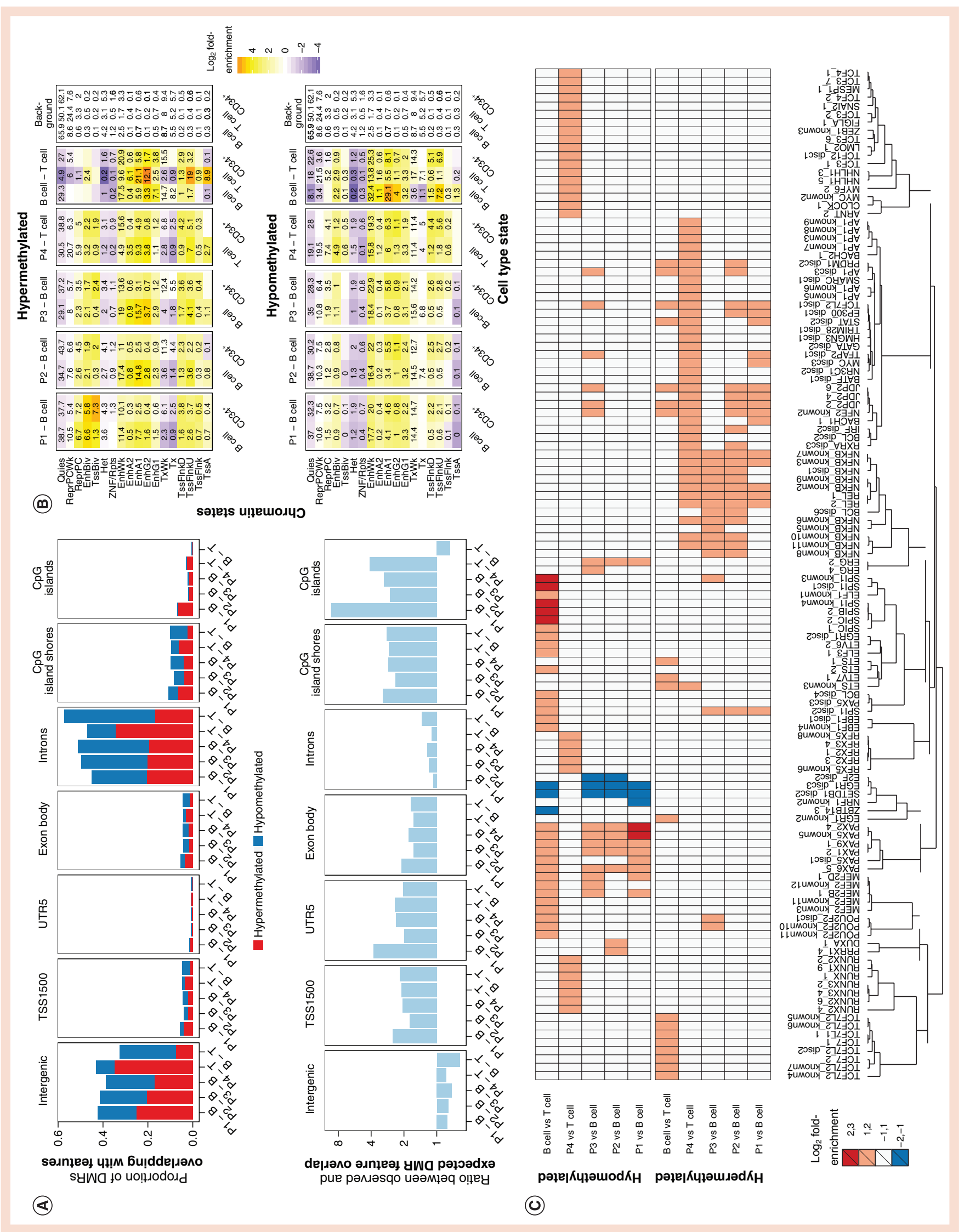


Figure 5. Annotation of differentially methylated regions in acute lymphoblastic leukemia patient cells and control cells (see facing page). (A) Proportion of hyper-methylated (red) and hypomethylated (blue) DMRs over-lapping with the genomic features indicated above the individual panels. The lower panel of figure A shows fold-enrichment of the base-overlap of DMRs with genomic features calculated against a sample-specific genomic background, which represents regions in the genome that can theoretically be called as a DMR. TSS1500, within 1500 bp of transcriptional start site; UTR5, 5' untranslated region; CpG islands; CpG island shores, \pm 2000 bp regions flanking CpG islands. (B) Enrichment of DMRs to chromatin states in ALL cells. The DMRs were intersected with chromatin state annotations from B cells, T cells and CD34+ cells, and the fold-enrichment was calculated from the observed base overlap with states divided by the genome-wide expected fraction of each state. The color key indicates $\log _{2}$ fold-enrichment and the numbers in the cells correspond to the proportion of overlapping DMR bases. Background indicates the proportion of each chromatin state in B cells, T cells and CD34+ cells. Empty cells represent nonsignificant fold-change differences (empiric randomization test; $p>0.05$ ). The upper panel shows enrichment of hyper-methylated DMRs, and the lower panel shows enrichment of hypomethylated DMRs in ALL cells. (C) Enrichment of transcription factor binding motifs in DMRs. Columns represent sequence motifs that are enriched more than twofold according to the color key in the lower left corner of panel $\mathrm{C}$ in at least one sample. Rows represent the comparison performed to define hyper- and hypomethylated DMRs as indicated on the left side of panel C. Transcription factor binding motifs (columns) were sorted based on hierarchical clustering of motif sequence similarity (lower part of figure).

ALL: Acute lymphoblastic leukemia; DMR: Differentially methylated region; TSS: Transcriptional start site.

those marks (Figure $8 \mathrm{C}$ ). According to RNA sequence data, the majority $(79 \%)$ of genes that are proximal to differentially methylated CpG islands with intermediate methylation levels are not expressed in the ALL cells or in the control cells, which is in sharp contrast with genes near unmethylated $\mathrm{CpG}$ islands, where the majority $(78 \%)$ of the genes are expressed (Figure 8D; Supplementary Figure 9).

To clarify the origin of intermediate DNA methylation at $\mathrm{CpG}$ islands in ALL patients, we examined the DNA methylation patterns along the individual sequence reads, each of which originates from an individual ALL cell, using a concept similar to that presented by Landau et al. [48] and by Landan et al. [49]. As illustrated (Figure 9A), intermediate DNA methylation levels can occur as a consequence of admixture of cells with different $\mathrm{CpG}$ site methylation patterns between individual sequence reads, but with concordant methylation levels for CpG sites along each read. Alternatively, the intermediate methylation levels may arise from admixture of ALL cells with variable and discordant methylation states along the sequence reads. These two mechanisms can be distinguished with the aid of the individual DNA sequence reads that correspond to individual DNA molecules that originate from a single cell, by calculating the proportion of discordantly methylated reads, in combination with the methylation variance between the DNA sequence reads across CpG islands (see 'Materials \& methods' section).

According to the combined analysis of these two metrics, only a minority of the $\mathrm{CpG}$ islands have concordant methylation levels along the sequence reads in combination with a large variance in DNA methylation between the sequence reads. These rare DNA methylation patterns at CpG islands could be the results of concordant clonal methylation differences like allele-specific methylation, or they could represent imprinted genomic regions. Out of the total $8204 \mathrm{CpG}$ islands with intermediate and variable DNA methylation levels we identified only $101 \mathrm{CpG}$ islands with less than $50 \%$ discordant sequence reads and a variance between the sequence reads that was larger than $12.5 \%$ that are shared between at least two of the ALL samples (Supplementary Table 6). Gene ontology analysis of genes associated with such CpG islands revealed a 28 -fold enrichment (hypergeometric test; $\mathrm{p}=8.9 \times 10^{-10}$ ) to known imprinted loci (Supplementary Table 7).

The by far largest part of the variability in DNA methylation appears to be the result of admixture of ALL cells with discordant methylation states along the chromosomes (Figure 9B). As can be seen by the grey areas in Figure $9 \mathrm{~B}$ more than $71 \%$ of the $\mathrm{CpG}$ islands have discordant methylation patterns in more than half of the sequence reads in the four ALL samples (P1-P4). Thus we conclude that the increased DNA methylation and large variability in $\mathrm{CpG}$ islands in ALL cells is a result of an apparent stochastic methylation process leading to discordant DNA methylation between ALL chromosomes.

\section{Discussion}

Our analysis of the DNA methylome in primary pediatric ALL cells from patients with four different cytogenetic backgrounds suggests that the global DNA methylation pattern in ALL is similar to that in a typical mammalian cell [15]. DNA methylation does not generally occur in $\mathrm{CpG}$-rich promoter regions, whereas regions with high methylation levels are found in genes and intergenic regions that are frequently interrupted by short stretches of hypomethylated CpG sites in lowdensity $\mathrm{CpG}$ regions, commonly overlapping known enhancer regions. Concurrent leukemia-specific differential methylation between the ALL cells and control cells primarily resides in CpG islands. 
(A)

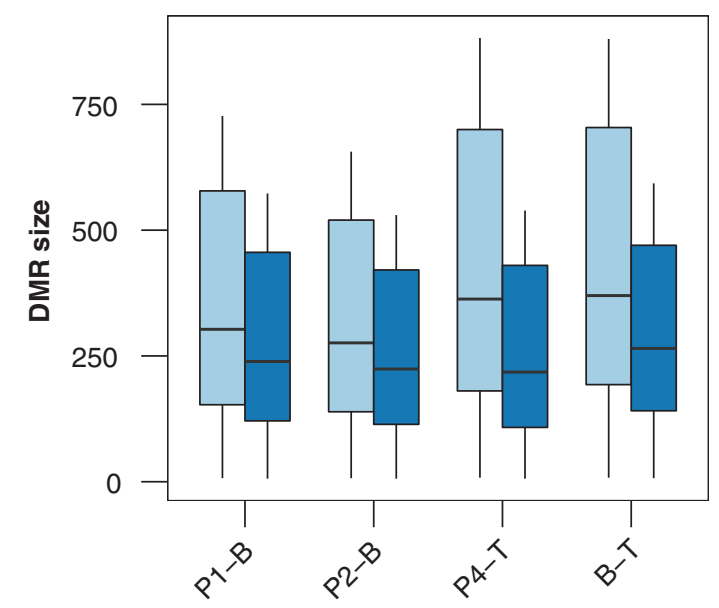

(C)

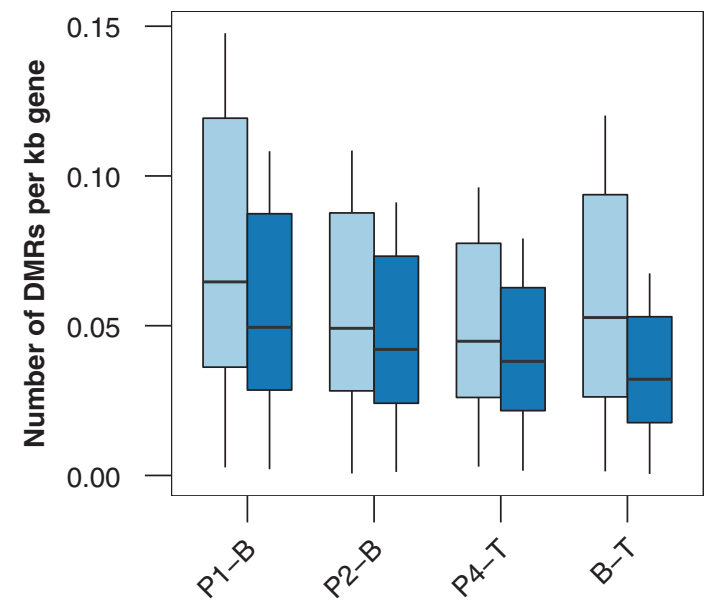

(B)

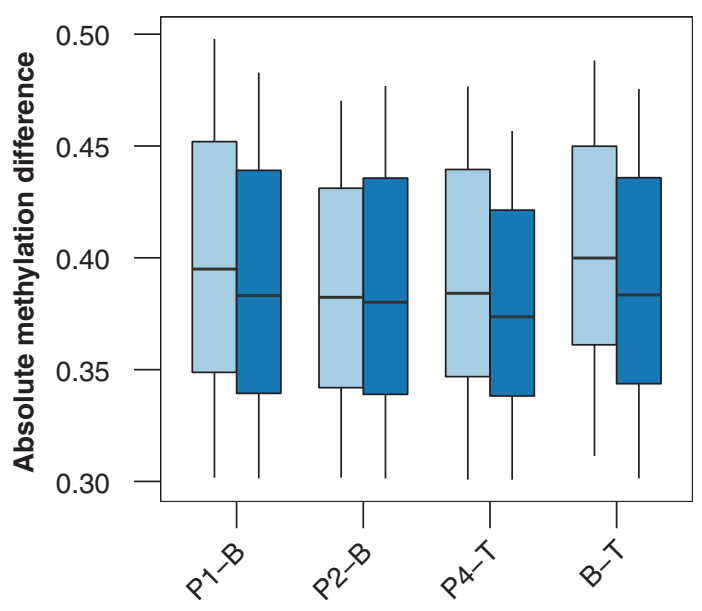

(D)

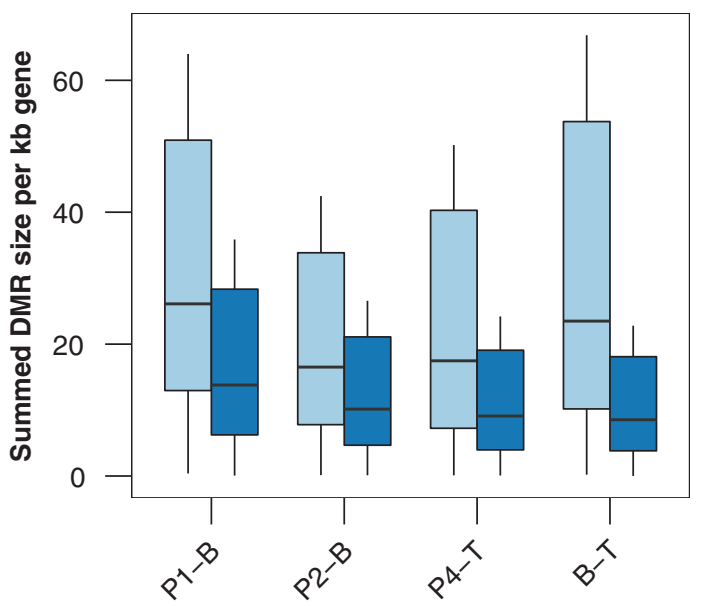

DE

Not DE

Figure 6. Differentially methylated regions associated with differentially expressed genes. (A-D) Light blue boxes show data for DMRs that are associated with DE, dark blue boxes show DMRs associated with genes that do not show differential expression (not DE). The horizontal bars in the boxes indicate median values. The DMRs were assigned to a protein-coding transcript if a DMR was located within $10 \mathrm{~kb}$ upstream of a transcription start site to $5 \mathrm{~kb}$ downstream of a protein-coding transcript. The cell comparisons to define DMRs are indicated along the horizontal axis below each pair of boxes (P1, P2 and P3 are acute lymphoblastic leukemia cells, B and T are control cells). (A) Box-plot of the distribution of the size (bp) of DMRs. (B) Box-plot of absolute DNA methylation level differences for individual DMRs. (C) Box-plot of the number of DMRs normalized to per kb of gene. (D) Box-plot of the summed DMR sizes normalized per kb of gene.

DE: Differentially expressed gene; DMR: Differentially methylated region.

Many of the DMRs that we discovered in ALL cells by comparison to mature B or T cells, are most likely not de novo acquired ALL-specific changes, but are shared between ALL cells and CD $34^{+}$cells. Thus the ALL cells may maintain an epigenetic signature from an ancestral developmental stage, or alternatively the ALL cells could have regained methylation pattern similar to those of undifferentiated progenitor cells. Part of the DNA methylation differences between ALL cells and control cells could arise as a consequence of clonal expansion of a single ALL cell leading to drift and fixation of intra-individual DNA methylation variations. Such variation caused by 'epigenetic drift' could also account for a proportion of the DMRs. Further analysis using WGBS of lymphoid progenitor cells in key developmental stages of hematopoiesis could aid to distinguish de novo ALL-specific methylation changes from methylation patterns in ALL that repre- 
sent a signature of ancestral cells and could reveal the origin of the cell type that the ALL developed from. Inclusion of WGBS data from lymphoid progenitor cells would have strengthened our study, as it would have added valuable information about the stages of transformation from normal cells into ALL cells of different subtype. Unfortunately, at the early stage for WGBS when our experiments were performed it was not possible to obtain a sufficient amount of lymphoid progenitor cells for extraction of the amount of DNA required for WGBS.

The majority of the DMRs discovered in our study are not directly associated with differentially expressed genes, although we observe an enrichment of DMRs to differentially expressed genes, compared with genes that do not show differential expression in our analysis. Across all comparisons we observed that DMRs that are co-localized with differentially expressed genes are on average larger and display larger absolute differences in methylation levels than DMRs that are not associated with differential expression. These findings could reflect active binding of TFs, whereas for DMRs associated with genes without differential expression, the DNA methylation differences could gradually disappear due to lack of binding of a TF, which would result in a reduced DMR size and diminishing DNA methylation. The TERF2 gene which contains two adjacent large DMR that

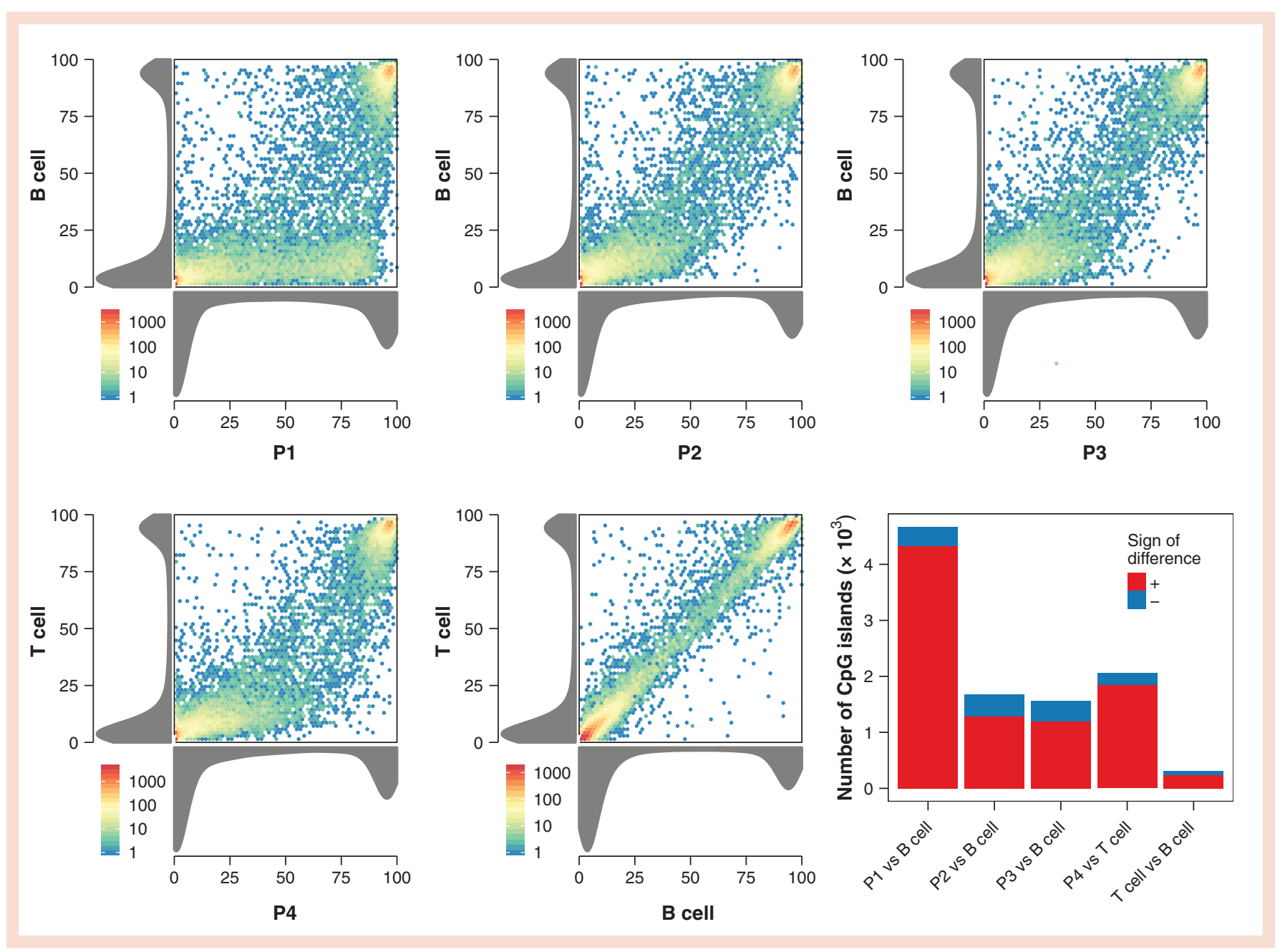

Figure 7. Aberrant DNA methylation of $\mathrm{CpG}$ islands in acute lymphoblastic leukemia cells. Average methylation levels in acute lymphoblastic leukemia (ALL) cells were plotted against those in control cells for each annotated CpG island. The grey areas below and on the left side of the panels show the density distributions of CpG island DNA methylation levels per sample. The B-cell precursor-ALL samples were compared against DNA methylation data from B cells, and the T-ALL sample was compared against DNA methylation data from $T$ cells as indicated on the left and below the panels. The color code in the scale bar shows number of observations at each position in the plot. The number of $\mathrm{CpG}$ islands with differential methylation between cell types is shown in the lower right panel. The horizontal axis indicates the pair-wise comparisons and the vertical axis indicates the number of $\mathrm{CpG}$ island with greater than $\pm 20 \%$ difference in average methylation levels in the pair-wise comparisons. Red color of the bar indicates increased methylation and blue indicates decreased methylation in ALL cells. 
(A)
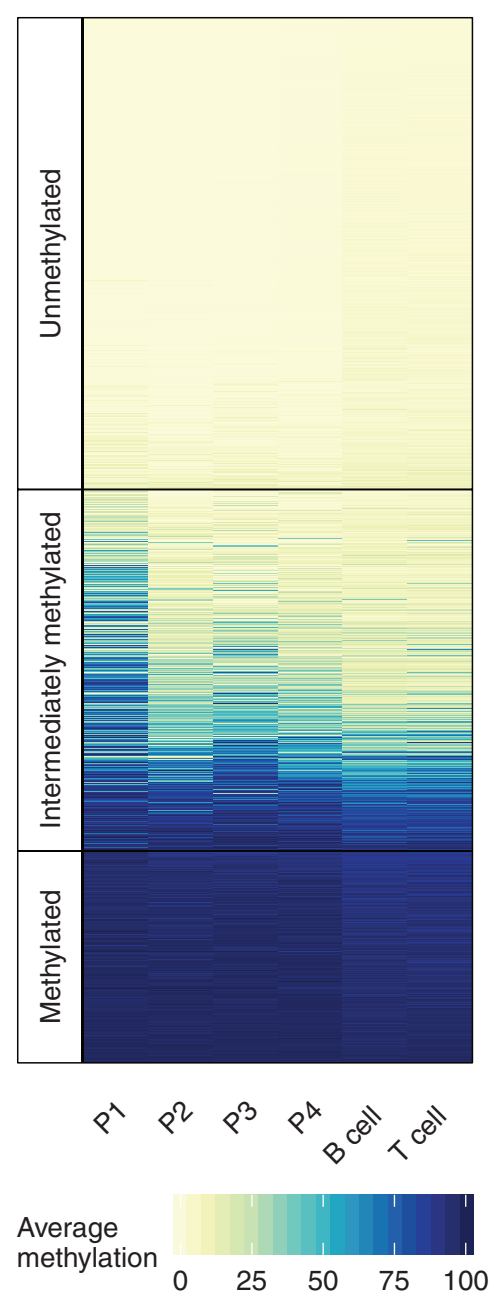

(B)

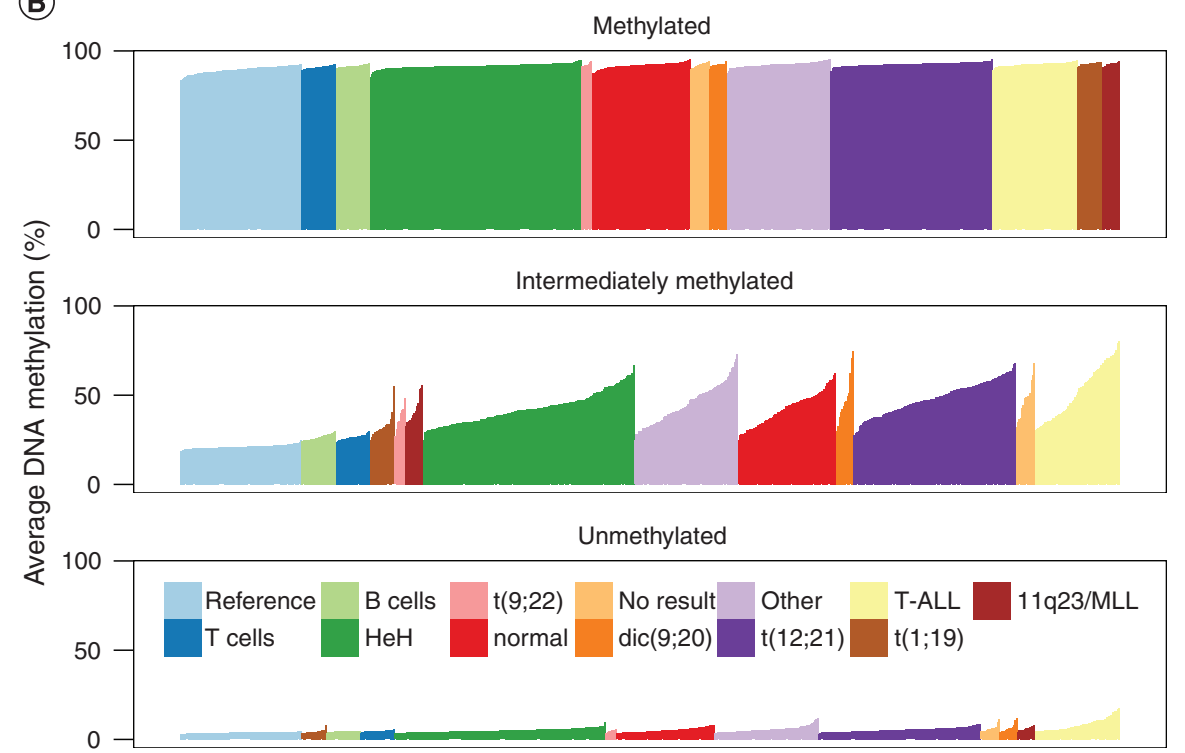

(C)
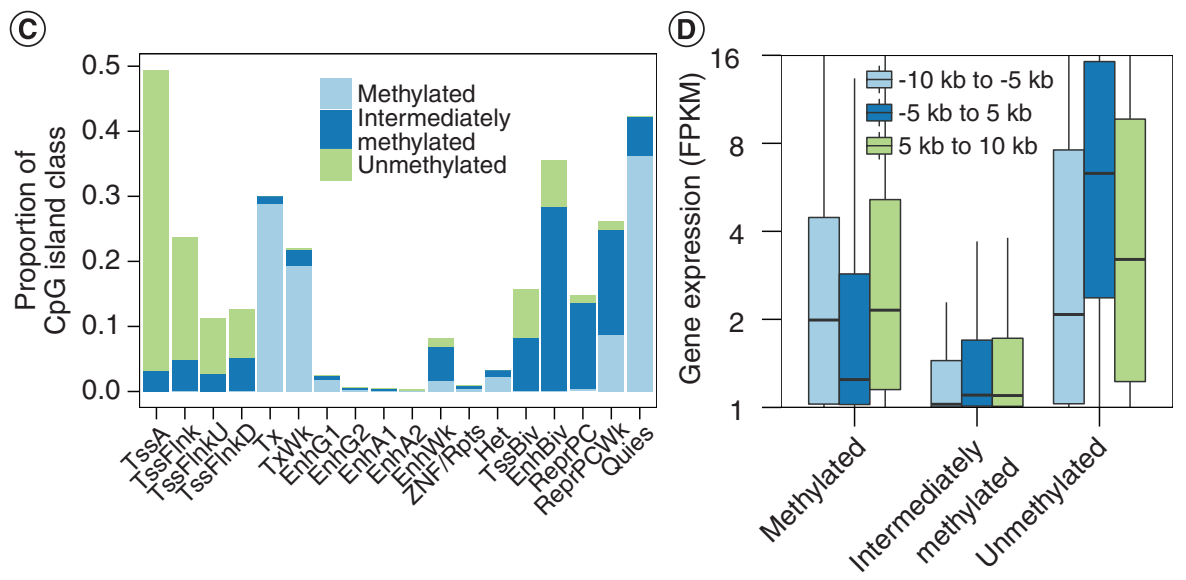

Figure 8. Overall DNA methylation patterns in CpG islands. (A) Heat map displaying the methylation levels for approximately $28,000 \mathrm{CpG}$ islands in acute lymphoblastic leukemia (ALL) cells and control cells. CpG islands (rows) partitioned into three groups (Methylated, Intermediately methylated and Unmethylated) based on their average methylation levels as indicated in the color key below panel A. Data have been sorted according to the DNA methylation levels in the CpG islands. (B) Confirmation of CpG island methylation levels using 450k DNA methylation BeadArray data from a large cohort of ALL samples and control cells. CpG sites were partitioned into three groups (Methylated, Intermediately methylated and Unmethylated) based on data from whole-genome bisulfite sequencing. Average genome-wide DNA methylation levels are shown on the y-axis for the individual samples. Samples within each ALL subtype, indicated by the color-codes, are sorted according to their DNA methylation levels. (C) Overlap of CpG islands classified as methylated, intermediately methylated or unmethylated according to their overlap with $\mathrm{B}$-cell chromatin states. Chromatin states are given on the $\mathrm{x}$-axis and the proportion of overlap for the three categories of CpG islands is color-coded as indicted by the color key in panel C. (D) Expression patterns of genes that are co-localized with CpG islands classified as either methylated, intermediately methylated or unmethylated. CpG islands were stratified into groups according to methylation status (methylated, intermediately methylated and unmethylated) and their distance from the nearest transcription start site as shown by the color-coded panel. Gene-expression levels (fragments per transcript per kilobase per million) for each gene associated with a CpG island were pooled for ALL samples P1-P4. Data from individual ALL samples and control cells can be found in Supplementary Material, Supplementary Figure 8.

are associated with strong differential expression of TERF2 in $\mathrm{t}(12 ; 21)$ ETV6-RUNX1 ALL exemplifies such a functional DMR. We also noted an increased number of DMRs per gene that are associated with differentially expressed genes, suggesting that mul- tiple active enhancers are required to significantly influence gene expression.

In normal cells, promoter $\mathrm{CpG}$ islands are hypomethylated with limited variation between cell types, similarly to what we observe for B and T cells (Figure 7). 
(A)

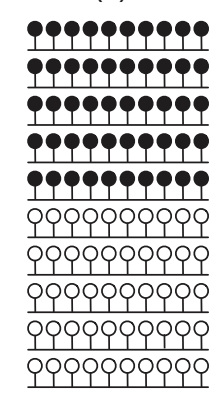

(b)

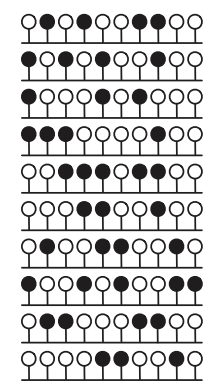

(c)

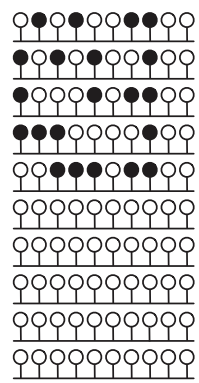

(d)

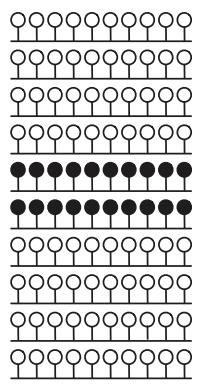

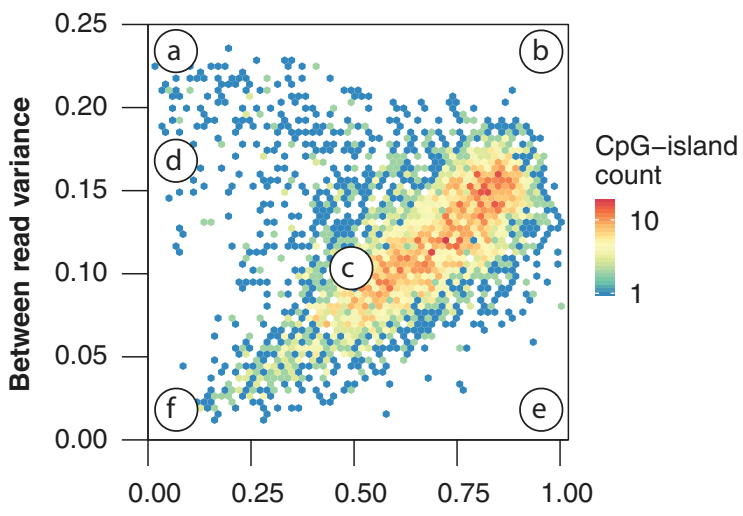

(e)

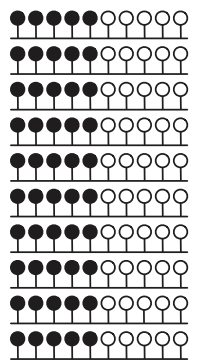

Proportion of discordant reads

(e)

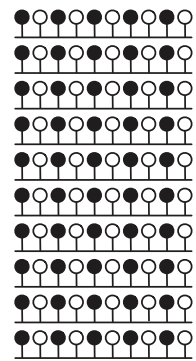

(f)

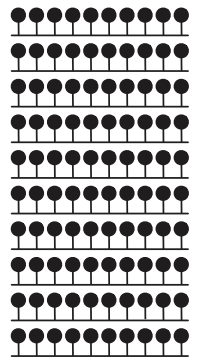

(f)

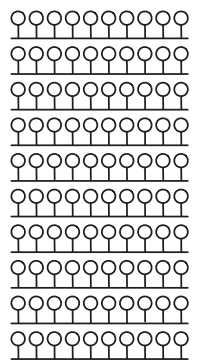

Figure 9. DNA methylation patterns in sequence reads from CpG islands with intermediate DNA methylation levels. (A) Theoretical illustration of alternative DNA methylation patterns in CpG islands with intermediate methylation levels. Empty and filled circles (a-e) represent unmethylated and methylated CpG sites along individual chromosomes. The graph shows the variance between genomic positions at each CpG site calculated across individual $\mathrm{CpG}$ islands on the $\mathrm{y}$-axis, plotted against the proportion of discordant reads identified within CpG islands on the $x$-axis. The encircled letters a-e in the plot represent the approximate positions at which the methylation patterns illustrated in a-e would be positioned. (a) A CpG island that shows allele-specific methylation or is an imprinted locus because the individual chromosomes are either completely methylated or unmethylated and observed at equal proportions. The variance within a position is at its maximum at 0.25 and the proportion of discordant reads would be zero. (b) A CpG island where all chromosomes show disordered DNA methylation. Both the discordance rate and the variance within a position are equal to 1.0. (c) Equal distribution of unmethylated and randomly methylated reads. (d) $80 \%$ unmethylated and $20 \%$ methylated reads. (e) Two examples of different DNA methylation patterns with the same position in the graph. (f) Examples of either completely methylated or unmethylated CpG sites.

Hyper-methylation of promoter CpG islands in tumor suppressor genes has been frequently observed [50,51], and it has been postulated that hyper-methylation would contribute to tumor progression by silencing tumor suppressor genes. This concept was challenged in a recent study [52], which showed that in seven cancer types, genes associated with hyper-methylated CpG islands are already silenced in the corresponding normal cells, making it unlikely that hyper-methylation per se would induce gene silencing and drive tumor 


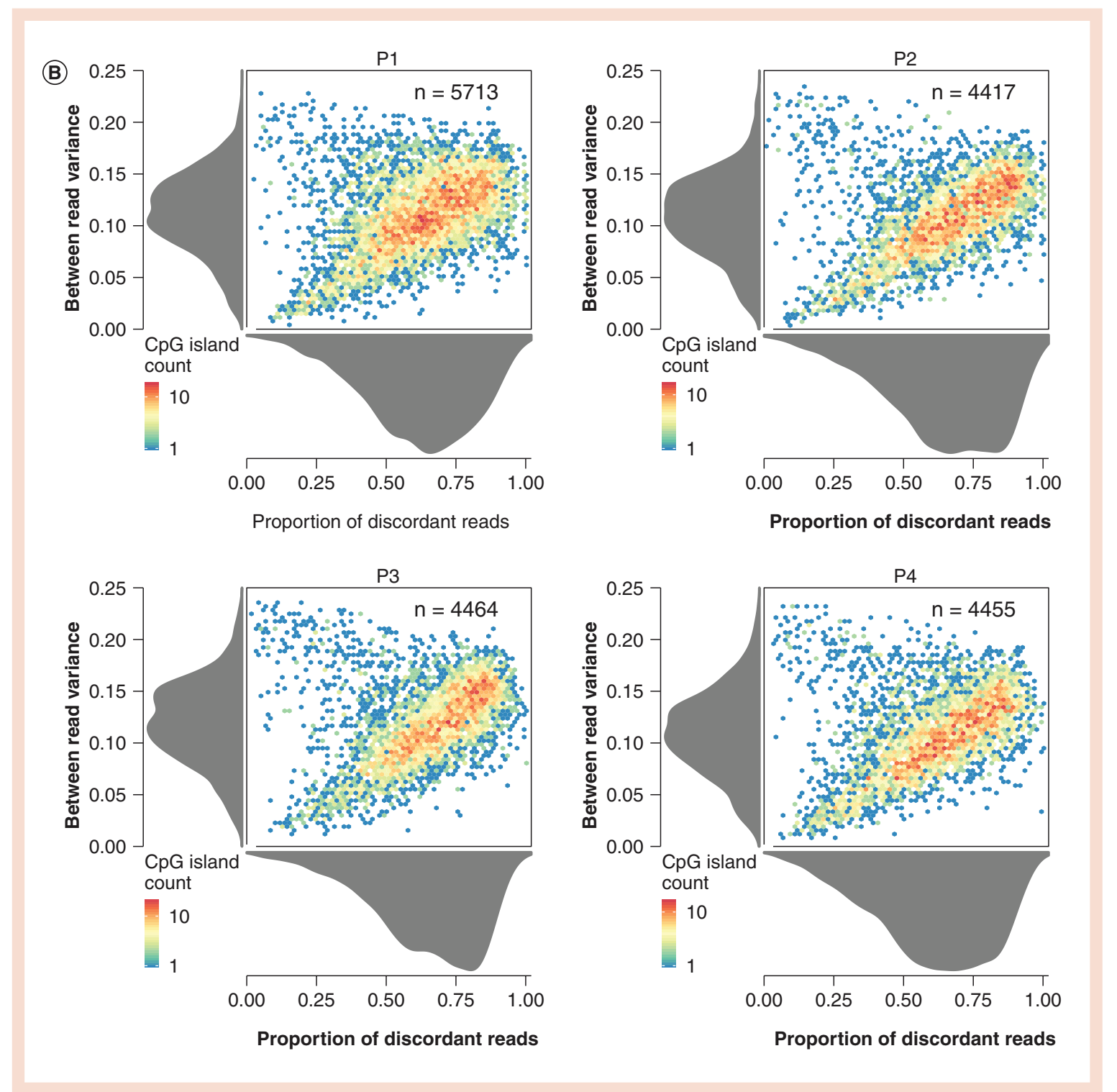

Figure 9. DNA methylation patterns in sequence reads from $\mathrm{CpG}$ islands with intermediate DNA methylation levels (cont.). (B) The graphs show the variance of DNA methylation calculated within each CpG site summed across individual intermediately methylated $\mathrm{CpG}$ islands on the $y$-axis, plotted against the proportion of discordantly DNA methylated read-pairs identified within the CpG island on the x-axis. A discordant DNA methylated read-pair is defined as a read-pair with less than $90 \%$ of its $\mathrm{CpG}$ sites either methylated or unmethylated. P1, P2, P3 and P4 above each plot indicate ALL patient names. The number of analyzed CpG islands is shown within each figure. The color code in the scale bar shows number of observations at each position in the plot. The grey areas below and on the left side of the panels show the density distributions of discordant reads and methylation variance between the DNA sequence reads across $\mathrm{CpG}$ islands, respectively.

progression. In the current study we found between 2000 and $4000 \mathrm{CpG}$ islands with increased DNA methylation in ALL cells, which is in contrast with the low number $(\mathrm{n}=\sim 350)$ of differentially methylated CpG islands that we detected compared with normal $\mathrm{B}$ and $\mathrm{T}$ cells. In aberrantly methylated $\mathrm{CpG}$ islands we observe highly variable, intermediate DNA methylation levels, while we find few examples of concurrently hyper-methylated $\mathrm{CpG}$ islands. Moreover, $\mathrm{CpG}$ islands that are intermediately methylated in ALL cells display increased DNA methylation in normal B and $\mathrm{T}$ cells, suggesting that methylation of $\mathrm{CpG}$ islands is not an exclusively ALL-specific feature. The molecular mechanisms by which the leukemic cells accumulate methylation marks at specific $\mathrm{CpG}$ islands warrant further investigation. It could be that the increased number of cell divisions and increased replication rate in cancer cells induce increased DNA methylation. Our 
data also show that genes that are associated with aberrant, intermediate DNA methylation levels are generally not expressed in the ALL cells nor in B and T cells, which argues against a role of increased methylation in $\mathrm{CpG}$ islands as a universal mechanism to shut down expression of tumor suppressor genes in ALL.

It is an interesting question how the pattern of intermediately methylated $\mathrm{CpG}$ islands arises on the molecular level. Bisulfite sequencing has the advantage that each sequence read is derived from a single chromosome, which allows investigation and quantification of intra-tumor DNA methylation patterns [49]. In our analysis, we observed that the bulk of the intermediately methylated $\mathrm{CpG}$ islands arise as a consequence of intra-chromosomal variability of DNA methylation. Previously published results from a comprehensive study in chronic lymphocytic leukemia (CLL) patients [48] used reduced representation bisulfite sequencing to assess the intra-chromosomal structure of DNA methylation by analyzing the methylation states at four consecutive $\mathrm{CpG}$ sites. In concordance with our results, the bulk of the heterogeneity of DNA methylation in CLL originates from an apparently stochastic process generating variability within chromosomes. The CLL study and a similar study of diffuse large B cell lymphoma tumors showed that despite the stochastic nature by which the methylation pattern arises, the increased intra-tumor heterogeneity in promoter regions is associated with adverse clinical outcome [53]. This finding suggests that enhanced epigenetic diversity could act as fuel for Darwinian selection, analogous to selection on genetic diversity that could promote malignant tumor development and resistance to treatment. Further research on intra-tumor heterogeneity of DNA methylation is warranted to fully comprehend the functional consequences and implications for treatment.

\section{Conclusion}

To date, the majority of DNA methylation studies have relied on data from array-based and targeted DNA methylation assays. We used unbiased WGBS to acquire near complete genome-wide information of DNA methylation patterns in ALL cells. We detected a large set of DMRs in ALL cells that are enriched to functional genomic regions, such as TF binding sites and hence could be important for ALL development and progression. The majority of DMRs that we identified are most likely not de novo acquired leukemiaspecific changes, but represents DNA methylation marks from previous cell developmental stages. Importantly, DNA methylation information can be valuable to determine the origin of the ALL cell. Our results also highlight that there is extensively increased DNA methylation in $\mathrm{CpG}$ islands and that intermediate and highly variable DNA methylation levels appear to arise as a consequence of intrachromosomal variability and not by concordant clonal methylation.

\section{Supplementary data}

To view the supplementary data that accompany this paper, please visit the journal website at: www.futuremedicine.com/ doi/full/10.2217/epi-2016-0052

\section{Author contributions}

AC Syvänen, G Lönnerholm, P Wahlberg conceived the study; P Wahlberg, J Nordlund, A Raine performed experiments; P Wahlberg, A Lundmark, J Nordlund analyzed the data; S Busche, T Pastinen, D Sinnett provided data; E Forestier, G önnerholm provided clinical material and data; K Tandre, L Rönnblom provided control cells; and P Wahlberg, A Lundmark, J Nordlund, AC Syvänen wrote the manuscript. All authors read and approved the manuscript.

\section{Acknowledgements}

The authors thank our colleagues in NOPHO and the ALL patients who contributed samples to the study.

\section{Financial \& competing interests disclosure}

This work was supported by grants from the Swedish Foundation for Strategic Research (RBC08-008; ACS, GL), the Swedish Cancer Society (140581; ACS), the Swedish Childhood Cancer Foundation (PR2014-0100; ACS), the Swedish Research Council for Science and Technology (VR-NT, C0524801; ACS) and joint funding from the Swedish Research Councils FORTE, FORMAS, VINNOVA, and VR (259-2012-23; ACS) for epigenetics. Bisulfite sequencing and RNA sequencing were performed by the SNP\&SEQ Technology Platform in Uppsala, which is part of the National Genomics Infrastructure hosted by Science for Life Laboratory. Computational analysis was performed using resources provided by the Swedish National Infrastructure for Computing (SNIC) through the Uppsala Multidisciplinary Center for Advanced Computational Science (UPPMAX). The authors have no other relevant affiliations or financial involvement with any organization or entity with a financial interest in or financial conflict with the subject matter or materials discussed in the manuscript apart from those disclosed.

No writing assistance was utilized in the production of this manuscript.

\section{Data deposition}

The DNA methylation data and RNA-sequencing results included in this publication have been deposited in NCBI's Gene Expression Omnibus (GEO) repository (GSE76270).

\section{Ethical conduct of research}

The authors state that they have obtained appropriate institutional review board approval or have followed the principles 
outlined in the Declaration of Helsinki for all human or animal experimental investigations. In addition, for investigations involving human subjects, informed consent has been obtained from the participants involved.

\section{Open access}

This work is licensed under the Creative Commons Attribution 4.0 License. To view a copy of this license, visit http:// creativecommons.org/licenses/by-nc-nd/4.0/

\section{Executive summary}

- We employed whole-genome bisulfite sequencing to generate complete high-resolution maps of the DNA methylomes of primary cells from patients with pediatric acute lymphoblastic leukemia (ALL).

- Compared with normal human B and T cells, whole-genome bisulfite sequencing identified thousands of differentially methylated regions (DMRs) in the ALL cells from four patients of different cytogenetic subtype. The DMRs are aggregated to regions of low CpG density and are enriched to gene regions and functional genomic elements, such as enhancers and known leukocyte-specific transcription factor binding sites.

- According to RNA-sequencing the DMRs in the ALL cells that are associated with differentially expressed genes have an increased size, a larger difference in absolute methylation levels and an increased number of DMRs than DMRs that do not overlap with differentially expressed genes.

- Hyper-methylated DMRs are enriched to CpG islands and genes located nearby these CpG islands are usually not expressed in ALL nor in control B and T cells. This finding indicates that hyper-methylation of CpG islands is unlikely to play a functional role in promoting cancer development by turning off the expression of tumor suppressor genes, contrary to what has been previously believed.

- The sequence reads from bisulfite-treated DNA allowed us to characterize the DNA methylation patterns along chromosomes that originate from individual ALL cells. According to this analysis the highly variable $C p G$ island methylation that we observe in the ALL cells is a consequence of random methylation of CpG sites.

\section{References}

1 Smith ZD, Meissner A. DNA methylation: roles in mammalian development. Nat. Rev. Genet. 14, 204-220 (2013).

2 Timp W, Feinberg AP. Cancer as a dysregulated epigenome allowing cellular growth advantage at the expense of the host. Nat. Rev. Cancer 13(7), 497-510 (2013).

3 Ley TJ, Ding L, Walter MJ et al. DNMT3A mutations in acute myeloid leukemia. N. Engl. J. Med. 363(25), 2424-2433 (2010).

4 Figueroa ME, Abdel-Wahab O, Lu C et al. Leukemic IDHI and $I D H 2$ mutations result in a hypermethylation phenotype, disrupt TET2 function, and impair hematopoietic differentiation. Cancer Cell 18(6), 553-567 (2010).

5 Inaba H, Greaves M, Mullighan CG. Acute lymphoblastic leukaemia. Lancet 381(9881), 1943-1955 (2013).

6 Nordlund J, Backlin CL, Wahlberg P et al. Genome-wide signatures of differential DNA methylation in pediatric acute lymphoblastic leukemia. Genome Biol. 14(9), r105 (2013).

7 Figueroa ME, Chen S-C, Andersson AK et al. Integrated genetic and epigenetic analysis of childhood acute lymphoblastic leukemia. J. Clin. Invest. 123, 3099-3111 (2013).

8 Hogan LE, Meyer JA, Yang J et al. Integrated genomic analysis of relapsed childhood acute lymphoblastic leukemia reveals therapeutic strategies. Blood 118(19), 5218-5226 (2011).

9 Borssen M, Palmqvist L, Karrman K et al. Promoter DNA methylation pattern identifies prognostic subgroups in childhood T-cell acute lymphoblastic leukemia. PLoS ONE 8(6), e65373 (2013).

10 Milani L, Lundmark A, Kiialainen A et al. DNA methylation for subtype classification and prediction of treatment outcome in patients with childhood acute lymphoblastic leukemia. Blood 115(6), 1214-1225 (2010).

11 Esteller M. CpG island hypermethylation and tumor suppressor genes: a booming present, a brighter future. Oncogene 21(35), 5427-5440 (2002).

12 Issa JP. CpG island methylator phenotype in cancer. Nat. Rev. Cancer 4(12), 988-993 (2004).

13 Stadler MB, Murr R, Burger L et al. DNA-binding factors shape the mouse methylome at distal regulatory regions. Nature 480, 490-495 (2011).

14 Ziller MJ, Gu H, Müller F et al. Charting a dynamic DNA methylation landscape of the human genome. Nature 500 (7463), 477-481 (2013).

15 Lister R, Pelizzola M, Dowen RH et al. Human DNA methylomes at base resolution show widespread epigenomic differences. Nature 462, 315-322 (2009).

16 Burger L, Gaidatzis D, Schubeler D, Stadler MB. Identification of active regulatory regions from DNA methylation data. Nucleic Acids Res. 41(16), e155 (2013).

17 Hovestadt V, Jones DTW, Picelli S et al. Decoding the regulatory landscape of medulloblastoma using DNA methylation sequencing. Nature 510, 537-541 (2014).

18 Schmiegelow K, Forestier E, Hellebostad M et al. Longterm results of NOPHO ALL-92 and ALL-2000 studies of childhood acute lymphoblastic leukemia. Leukemia 24(2), 345-354 (2010).

19 Lindqvist CM, Nordlund J, Ekman D et al. The mutational landscape in pediatric acute lymphoblastic leukemia deciphered by whole genome sequencing. Hum. Mutat. 36(1), 118-128 (2015).

20 Healy J, Belanger H, Beaulieu P, Lariviere M, Labuda D, Sinnett D. Promoter SNPs in G1/S checkpoint regulators and their impact on the susceptibility to childhood leukemia. Blood 109(2), 683-692 (2007). 
21 Johnson MD, Mueller M, Game L, Aitman TJ. Single nucleotide analysis of cytosine methylation by wholegenome shotgun bisulfite sequencing. In: Current Protocols in Molecular Biology. John Wiley \& Sons, Inc. (2001).

22 Martin M. Cutadapt removes adapter sequences from highthroughput sequencing reads. EMBnet 17(1), doi:10.14806/ ej.17.1.200 (2011).

23 Li H, Durbin R. Fast and accurate long-read alignment with Burrows-Wheeler transform. Bioinformatics 26(5), 589-595 (2010).

24 Picard Tools.

http://broadinstitute.github.io/picard/

25 Liu Y, Siegmund KD, Laird PW, Berman BP. Bis-SNP: combined DNA methylation and SNP calling for bisulfiteseq data. Genome Biol. 13, R61 (2012).

26 Hodges E, Molaro A, Dos Santos CO et al. Directional DNA methylation changes and complex intermediate states accompany lineage specificity in the adult hematopoietic compartment. Mol. Cell 44(1), 17-28 (2011).

27 Hansen KD, Langmead B, Irizarry RA. BSmooth: from whole genome bisulfite sequencing reads to differentially methylated regions. Genome Biol. 13, R83 (2012).

Quinlan AR, Hall IM. BEDTools: a flexible suite of utilities for comparing genomic features. Bioinformatics 26(6), 841-842 (2010).

29 Roadmap Epigenomics Project. www.roadmapepigenomics.org

30 Roadmap Epigenomics Project. http://egg2.wustl.edu/

31 ENCODE: Encyclopedia of DNA Elements. www.encodeproject.org

32 Kheradpour P, Kellis M. Systematic discovery and characterization of regulatory motifs in ENCODE TF binding experiments. Nucleic Acids Res. 42(5), 2976-2987 (2014).

33 Motif browser for ENCODE TF ChIP-seq datasets. http://compbio.mit.edu/encode-motifs/

34 Encode-motifs. http://compbio.mit.edu/encode-motifs/motifs-sim.txt.gz

35 Kim D, Pertea G, Trapnell C, Pimentel H, Kelley R, Salzberg SL. TopHat2: accurate alignment of transcriptomes in the presence of insertions, deletions and gene fusions. Genome Biol. 14(4), R36 (2013).

36 Liao Y, Smyth GK, Shi W. The Subread aligner: fast, accurate and scalable read mapping by seed-and-vote. Nucleic Acids Res. 41(10), e108 (2013).

37 Robinson MD, McCarthy DJ, Smyth GK. edgeR: a bioconductor package for differential expression analysis of digital gene expression data. Bioinformatics 26(1), 139-140 (2010).

38 Ritchie ME, Phipson B, Wu D et al. limma powers differential expression analyses for RNA-sequencing and microarray studies. Nucleic Acids Res. 43(7), e47 (2015).
39 McLean CY, Bristor D, Hiller M et al. GREAT improves functional interpretation of cis-regulatory regions. Nat. Biotechnol. 28(5), 495-501 (2010).

40 Berman BP, Weisenberger DJ, Aman JF et al. Regions of focal DNA hypermethylation and long-range hypomethylation in colorectal cancer coincide with nuclear lamina-associated domains. Nat. Genet. 44, 40-46 (2012).

41 Roadmap Epigenomics C, Kundaje A, Meuleman W et al. Integrative analysis of 111 reference human epigenomes. Nature 518(7539), 317-330 (2015).

42 Ernst J, Kellis M. ChromHMM: automating chromatin-state discovery and characterization. Nat. Methods 9(3), 215-216 (2012).

43 Biroccio A, Cherfils-Vicini J, Augereau A et al. TRF2 inhibits a cell-extrinsic pathway through which natural killer cells eliminate cancer cells. Nat. Cell Biol. 15, 818-828 (2013).

44 ENCODE: Encyclopedia of DNA Elements. www.encodeproject.org

45 Medvedovic J, Ebert A, Tagoh H, Busslinger M. Pax5: a master regulator of $\mathrm{B}$ cell development and leukemogenesis. Adv. Immunol. 111, 179-206 (2011).

46 Mullighan CG, Goorha S, Radtke I et al. Genome-wide analysis of genetic alterations in acute lymphoblastic leukaemia. Nature 446(7137), 758-764 (2007).

47 Gerondakis S, Siebenlist U. Roles of the NF-kappaB pathway in lymphocyte development and function. Cold Spring Harb. Perspect Biol. 2(5), a000182 (2010).

48 Landau Dan A, Clement K, Ziller Michael J et al. Locally disordered methylation forms the basis of intratumor methylome variation in chronic lymphocytic leukemia. Cancer Cell 26, 813-825 (2014).

49 Landan G, Cohen NM, Mukamel Z et al. Epigenetic polymorphism and the stochastic formation of differentially methylated regions in normal and cancerous tissues. Nat. Genet. 44, 1207-1214 (2012).

50 Greger V, Passarge E, Hopping W, Messmer E, Horsthemke B. Epigenetic changes may contribute to the formation and spontaneous regression of retinoblastoma. Hum. Genet. 83(2), 155-158 (1989).

51 Gonzalez-Zulueta M, Bender CM, Yang AS et al. Methylation of the 5' CpG island of the $p 16 / C D K N 2$ tumor suppressor gene in normal and transformed human tissues correlates with gene silencing. Cancer Res. 55(20), 4531-4535 (1995).

52 Sproul D, Kitchen RR, Nestor CE et al. Tissue of origin determines cancer-associated $\mathrm{CpG}$ island promoter hypermethylation patterns. Genome Biol. 13, R84 (2012).

53 Pan $\mathrm{H}$, Jiang Y, Boi $\mathrm{M}$ et al. Epigenomic evolution in diffuse large B-cell lymphomas. Nat. Commun. 6, 6921 (2015). 